May 30, 2018

UMD-PP-09-057, MADPH-09-1547, NPAC-09-14

\title{
LHC Signals for Coset Electroweak Gauge Bosons in Warped/Composite PGB Higgs Models
}

\author{
Kaustubh Agashe ${ }^{a}$, Aleksandr Azatov ${ }^{a}$, Tao $\operatorname{Han}^{b}$, Yingchuan Li ${ }^{b}$, Zong-Guo $\mathrm{Si}^{c}$, Lijun \\ $\mathrm{Zhu}^{a}$ \\ ${ }^{a}$ Maryland Center for Fundamental Physics, Department of Physics, University of Maryland, \\ College Park, MD 20742, U.S.A. \\ ${ }^{b}$ Department of Physics, University of Wisconsin, Madison, WI 53706, U.S.A \\ ${ }^{c}$ Department of Physics, Shandong University, Jinan Shandong 250100, China
}

\begin{abstract}
The framework of a warped extra dimension with the Standard Model (SM) fields propagating in it is a very well-motivated extension of the SM since it can address both the Planck-weak and flavor hierarchy problems of the SM. Within this framework, solution to the little hierarchy problem motivates extending the SM electroweak (EW) $5 D$ gauge symmetry in such a way that its breakdown to the SM delivers the SM Higgs boson. We study signals at the large hadron collider (LHC) for the extra EW (called coset) gauge bosons, a fundamental ingredient of this framework. The coset gauge bosons, due to their unique EW gauge quantum numbers [doublets of $S U(2)_{L}$ ], do not couple at leading order to two SM particles. We find that, using the associated production of the charged coset gauge bosons via their coupling to bottom quark and a (light) Kaluza-Klein excitation of the top quark, the LHC can have a $3 \sigma$ reach of $\sim 2(2.6) \mathrm{TeV}$ for the coset gauge boson masses with $\sim 100(1000) \mathrm{fb}^{-1}$ luminosity. Since current theoretical framework(s) suggest an indirect lower limit on coset gauge boson masses of $\gtrsim 3 \mathrm{TeV}$, luminosity or energy upgrade of LHC is likely to be crucial in observing these states.
\end{abstract}




\section{Introduction}

The framework of a warped extra dimension with Standard Model (SM) fields propagating in it [1, 2] can address both the Planck-weak and flavor hierarchy problems of the SM: for a review and further references, see [3]. The resolution of the Planck-weak hierarchy relies on the anti-de Sitter (AdS) geometry leading to exponential dependence of the effective $4 D$ mass scale (including UV cut-off) on the location in the extra dimension. In particular, this mass scale can be Planckian near one end of the extra dimension (called Planck brane), where the $4 D$ graviton is automatically localized thus accounting for the strength of gravity. On the other hand, the natural mass scale can be much smaller, for example, $O(\mathrm{TeV})$, near the other end (called the $\mathrm{TeV}$ brane) where the SM Higgs sector is localized. Thus, the Higgs mass scale is not sensitive to the Planck scale. Crucially, such a large hierarchy of mass scales at the two ends of the extra dimension can be achieved with only a modest proper size of the extra dimension in units of the AdS curvature radius.

However, with the $5 \mathrm{D}$ electroweak (EW) gauge symmetry being $S U(2)_{L} \times S U(2)_{R} 11$ and a Higgs transforming as a bi-doublet (henceforth called "minimal Higgs sector"), the framework still suffers from an incarnation of the little hierarchy problem. Namely, the Higgs mass is still sensitive to the $5 D$ cut-off, albeit warped-down (compared to the fundamental $5 D$ scale which is Planckian) at the TeV brane. The problem is that the mass scale of the Kaluza-Klein (KK) excitations of the $\mathrm{SM}$ particles is constrained to be at least a few $\mathrm{TeV}$ by various precision tests (see reference [3] for a review) and the (warped-down) $5 D$ cut-off should be larger than the KK scale by (roughly) an order-of-magnitude in order for the $5 D$ effective field theory description to be valid.

This naturalness problem motivates incorporating more structure in (i.e., a non-minimal) Higgs/EW sector. The idea is to suitably extend the $5 D$ EW gauge symmetry beyond the SM - the additional 5D EW gauge fields are called coset gauge bosons - and break it down to the $\mathrm{SM}$ by a scalar vev localized near $\mathrm{TeV}$ brane [5]. It can be shown that in this process, a massless (at tree-level) scalar mode (localized near the TeV brane) with SM Higgs quantum numbers can emerge. Moreover, the quantum corrections to the Higgs mass in this case has a reduced sensitivity to the $5 D$ cut-off.

In this paper, we begin a study of signals for coset gauge bosons in this framework at the large hadron collider (LHC). We find that the $3 \sigma$ reach of the LHC for the coset gauge bosons is $\sim 2.6$ $\mathrm{TeV}$ with $\sim 1000 \mathrm{fb}^{-1}$ of integrated luminosity, under certain well-motivated assumptions which we discuss. However, we also argue that the (indirect) lower bound on masses of coset gauge boson masses is expected to be (at least) $\sim 3 \mathrm{TeV}$ [6] (for review see reference [3]). So, our results provide a strong motivation for LHC luminosity and/or energy upgrade.

\footnotetext{
${ }^{1}$ Here, we include a $S U(2)_{R}$ factor, which is motivated by suppressing contributions to the $T$ parameter [4, as part of the "SM" EW gauge symmetry.
} 
An outline of our paper is as follows. We begin with an overview of the above framework which we call (in its full generality) "warped/composite PGB Higgs" for reasons which we explain there. Then, in section 3 we present a discussion of this framework using the convenient "twosite" approach [7] in order to get a general idea of spectrum and couplings of coset gauge bosons. In Section 4, we review specific warped extra dimensional models, namely, minimal "gauge-Higgs unification" (GHU) models, and the mechanism of radiative generation of Higgs potential. In particular, in section 4.4, we focus on the couplings of coset gauge boson in the GHU framework, showing in section 4.4.1 that the couplings of coset gauge bosons follow a general pattern which is independent of the details of this $5 \mathrm{D}$ model, and then in section 4.4 .2 presenting the exact formulae for them in the specific model within this framework by Medina et. al. [8. In Section 4.5, we show our numerical results for the particle spectrum and couplings from a scan of parameter space in the model (in the process backing-up our estimates for the couplings of the coset gauge bosons from section 4.4.1), and present sample points for collider study. Section 5 focuses on the collider phenomenology, where we study the production and decay of coset gauge bosons, and the prospect of their discovery at LHC. We conclude in Section 6. Technical details of the $5 D$ model are relegated to appendices.

\section{Overview}

As discussed in the introduction, we study the warped extra dimensional models where the SM Higgs arises from the breaking of an extended EW gauge symmetry down to the SM gauge symmetry near the $\mathrm{TeV}$ brane. A particular limit of this framework is where the scalar vev involved in this breaking of EW gauge symmetry is much larger than the AdS curvature scale such that the above breaking of $5 D \mathrm{EW}$ gauge symmetry is effectively the result of Dirichlet boundary condition on the $\mathrm{TeV}$ brane. The massless scalar mode can then be thought of as the extra polarization $\left(A_{z}\right)$ of the coset gauge fields. Hence, this model is dubbed "gauge-Higgs unification (GHU)": see, for example, reference [9] for a review of and more references for this idea. Quantum corrections do generate a potential (including a mass term) for it - this is the Hosotani mechanism for symmetry breaking [10. However, such effects are saturated at the typical KK scale rather than at the warped-down $5 D$ cut-off [5, 11, 12].

By the AdS/CFT correspondence [13], the general $5 D$ framework mentioned above [i.e., whether vev breaking $S O(5) \rightarrow S O(4)$ is infinite as in GHU or not] is conjectured to be a dual description of $(4 D)$ Georgi-Kaplan (GK) models [14]. In GK models, the SM Higgs is a composite of purely $4 D$ strong dynamics which is also a pseudo-Goldstone boson (PGB) of a spontaneously broken global symmetry and hence naturally lighter than the compositeness scale (dual to the typical KK scale) [5, 15]. This aspect of the $5 D$ models motivates using the terminology warped/composite PGB 
Higgs for this general framework, i.e., including various $5 D$ models [i.e., both the infinite scalar vev for $S O(5) \rightarrow S O(4)$ breaking, i.e. the GHU models, and the finite scalar vev] and $4 D$ models based on strong dynamics.

Our goal is to study how to distinguish the possibility of such a framework from the minimal Higgs sector framework by directly producing the extra particles (i.e., those arising as a result of the extension of the $5 D$ EW gauge symmetry) at the LHC2. Clearly, the $5 D$ fermions - whose zeromodes are identified with the SM fermions - must also now be in representations of the extended $5 D$ EW gauge symmetry, i.e., they are larger than in the case of minimal Higgs sector, with the extra components not having zero-modes (just like the coset gauge bosons). In particular, in some $5 D$ Warped/composite PGB Higgs models, some of these fermionic KK states (associated with top/bottom quarks) are lighter than SM gauge KK modes [6, 16, 8] (and hence, as discussed below, lighter than the coset gauge boson), whereas KK fermions have same mass as gauge KK modes in minimal Higgs sector framework. Hence these fermionic KK modes might be easier to detect at the LHC [17] than the SM (or coset) gauge KK modes and their discovery would be suggestive of warped/composite PGB Higgs models rather than the models with minimal Higgs sector. However, in the models constructed so far, most of these light fermionic states have the same quantum numbers under SM EW symmetry as those of SM fermions 3 so that they could be mistaken for similar states in other extensions of the SM. Thus, it is crucial to consider additional signals for the warped/composite PGB Higgs framework.

Such a test can be provided by detection of

- the coset gauge bosons which, being doublets of $S U(2)_{L}$, have novel (i.e., non-adjoint) representations under the SM EW gauge symmetry

(such quantum numbers for gauge bosons are obviously absent in the minimal Higgs sector framework). Thus these coset gauge bosons can result in distinctive LHC signals as compared to EW gauge KK modes in minimal Higgs sector models. Similarly, we discuss how coset gauge bosons can also be differentiated from new gauge bosons in other extensions of the SM. This feature of coset gauge bosons motivates our study in this paper of signals from their direct production at the

\footnotetext{
${ }^{2}$ Alternatively, one can probe the extra states indirectly, for example, via their virtual effects on lower-energy observables or how the properties of the usual states are modified in warped/composite PGB Higgs framework relative to minimal Higgs sector framework. However, such indirect effects might not be able to provide robust distinction between the two frameworks. The reason is that the minimal Higgs sector framework has a large number of free parameters and hence, for some choice of these, can mimic effects of extra particles of warped/composite PGB Higgs framework.

${ }^{3}$ The exception is a $5 / 3$-charged light KK fermion, but its existence might have more to do with the need for $Z b b$ protection rather than PGB Higgs.

${ }^{4} \mathrm{KK}$ fermionic states in the minimal Higgs sector framework also have the same quantum numbers, although these states are expected to be as heavy as SM gauge KK modes. So, there is less possibility of confusion between minimal Higgs sector models and warped/composite PGB Higgs framework based on these states.
} 
LHC 5

Our study suggests that

- the LHC $3 \sigma$ reach for (charged) coset gauge bosons masses is $\sim 2(2.6) \mathrm{TeV}$ with $\sim 100(1000)$ $\mathrm{fb}^{-1}$ luminosity, using their associated production with (light) KK top and decay into KK top and bottom quarks.

For this analysis, we use values of couplings which are motivated by the $(5 D)$ minimal (i.e., with no brane-localized kinetic terms for bulk fields and with $\mathrm{AdS}_{5}$ metric: see later) GHU model. A note on the allowed mass scale is in order here. In the minimal GHU model, it turns out that the coset gauge boson mass is $\approx 5 / 3$ larger than SM gauge KK modes [11]. And, the lower bound on the latter gauge boson masses is $\sim 3 \mathrm{TeV}$ from EW precision tests [6] (for a review see reference [3]), assuming custodial symmetries are implemented [4, 19] (and, depending on details of flavor structure, the bound can be somewhat stronger from flavor violation [20, 21] 6 although these constraints can be ameliorated by addition of $5 D$ flavor symmetries [24]). Thus, the coset gauge boson mass is constrained to be at least $5 \mathrm{TeV}$ which is well beyond reach of even 1000 $\mathrm{fb}^{-1}$ luminosity at the LHC 7 This situation then motivates upgrade of the energy of the LHC or building another higher-energy collider.

However, in non-minimal $5 D$ models - for example, with brane-localized kinetic terms for bulk fields [25] or with the metric near the TeV brane being modified from pure AdS [26] within the GHU models or with the scalar vev giving masses to coset gauge bosons being finite (instead of infinite as in GHU models), the indirect bound on coset mass scale might be relaxed because the ratio of coset to SM gauge KK masses is closer to 1. In fact, inspired by deconstruction/latticization and the AdS/CFT correspondence, a purely 4D, two-site approach [7] - keeping only SM and 1st KK excitations - has been proposed in order to efficiently/economically capture the phenomenology of similar variations of $5 D$ models with a minimal Higgs sector. Such a two-site approach can be extended to PGB Higgs models as well [27].

Using a two-site approach for the general warped/composite PGB Higgs, we argue that

- coset gauge bosons are expected to be at most be as light as (i.e., cannot be lighter than) SM gauge KK (or composite) modes.

\footnotetext{
${ }^{5}$ Very recently, in reference [18, a different signal (than what we study) for coset gauge bosons was suggested based (again) on the distinctive quantum numbers, but it was not studied in the context of a complete framework, for example, one that explains the flavor hierarchy.

${ }^{6}$ See references 22] and 23] for "latest" constraints from lepton and quark flavor violation, respectively, i.e.,including variations of the minimal framework.

${ }^{7}$ The bound on mass scale of coset gauge bosons from precision tests involving exchange of coset gauge bosons themselves is rather weak since there is no coupling of single coset gauge boson to purely SM particles at leading order (simply due to quantum numbers) so that coset gauge boson exchange at tree-level does not contribute to purely SM operators (and hence precision tests). The flip side of this feature is that resonant production of coset gauge bosons is suppressed, which is in part responsible for the poor LHC reach.
} 
Moreover, using the same approach, it can be shown that the bound on SM gauge KK (or composite) modes is unlikely to be reduced below $\sim 3 \mathrm{TeV}$ even in the non-minimal models, i.e., in the general framework 8 Thus, coset gauge bosons are expected to have mass $\gtrsim 3 \mathrm{TeV}$ in general. We argue based on the two-site approach that couplings of coset gauge bosons in the general framework will still be similar to those in minimal $5 D$ GHU models which we used for the study of LHC signals. This feature implies that the LHC reach for coset gauge bosons that we find based on couplings in minimal $5 D$ GHU model is expected to apply in general to the framework of warped/composite PGB Higgs. Thus, even optimistically, i.e., assuming that in some models within this framework the coset gauge bosons can be as light as SM gauge KK modes and using the $1000 \mathrm{fb}^{-1}$ luminosity, we see that the LHC can barely be sensitive to the lower (indirect) limit of $\sim 3 \mathrm{TeV}$ on coset gauge boson masses.

\section{Model-independent Analysis Using Two-site Approach}

In this section, we provide a rough description of masses and couplings of the coset gauge bosons of the general warped/composite PGB Higgs framework, i.e., the analysis presented here is applicable to both $5 D$ and $4 D$ models in this framework. The detailed description of a specific (5D) model, namely minimal GHU, will be given in the next section.

Here we use the two-site model [7] which is a convenient parametrization for this framework. It can be shown that this effective $4 \mathrm{D}$ description is the deconstructed version of warped extra dimension models with SM fields propagating in the bulk, including the zero and only the 1st KK modes. In the original setup presented in reference [7], Higgs was not a PGB. So first we will briefly review this model (for more details, the reader is referred to this paper), and then we will show what changes we have to make to account for the PGB origin of the Higgs.

The original two-site model consists of two sectors: "elementary" and "composite" (this nomenclature is inspired by the AdS/CFT correspondence). The elementary sector is a copy of all SM states except for the Higgs field. The composite sector consists of massive gauge bosons, massive vector-like fermions and the Higgs field. The composite sector states live in complete representation of the global symmetry $S U(3)_{c} \times S U(2)_{L} \times S U(2)_{R} \times U(1)_{X}$, where the additional custodial $S U(2)_{R}$ is introduced to suppress new physics contribution for $T$ parameter. The massive gauge bosons live in adjoint representation while part of massive fermions live in the same representation as that of SM fermion.

These two sectors mix with each other, leading to massless fermion and gauge boson eigenstates which correspond to SM fermions $\left(\psi_{L, R}\right)$ and gauge bosons $\left(A_{\mu}\right)$ before Electro-Weak Sym-

\footnotetext{
${ }^{8}$ It has been claimed that in soft-wall models, this bound can be lower than $3 \mathrm{TeV}$. However, such models have not been developed fully as yet.
} 
metry Breaking (EWSB). The heavy eigenstates are denoted by $\rho_{\mu}$ for gauge bosons and $\chi_{L, R}$ for fermions 9 The SM states (except for the Higgs) are mixtures of elementary and composite states:

$$
|\mathrm{SM}\rangle=\cos \theta \mid \text { elementary }\rangle+\sin \theta \mid \text { composite }\rangle,
$$

where all SM states (except for the top) are mostly made of the elementary sector ones (i.e., $\sin \theta \ll$ 1), while the heavy states are mostly the composite sector ones and finally the SM Higgs is fully a composite sector state. The composite sector states are assumed to have strong couplings to each other, in order to match the 5D description (or equivalently, inspired by AdS/CFT correspondence). We use $g_{*}$ and $Y_{*}$ to denote the composite gauge and Yukawa couplings (and will take them to be roughly of order a few). In the flavor anarchy models, $Y_{*}$ for different flavors are of the same order and have no structure, which we assume for the following discussion. Clearly the SM states couple to heavy states through the mixing (Eq. (1)). For example the Yukawa couplings between SM fermions and Higgs is given schematically by

$$
Y_{S M} \sim \sin \theta_{\psi_{L}} Y_{*} \sin \theta_{\psi_{R}}
$$

The fermionic mixing angles $\theta_{\psi_{L, R}}$ are assumed to be hierarchical, which explains the SM fermion mass hierarchy. In warped extra dimension picture, $\sin \theta_{\psi}$ is related to the fermion zero mode wavefunction evaluated at the $\mathrm{TeV}$ brane (see $f(c)$ in Eq. (19), with an exponential dependence on 5D mass parameter, $c$ ), thus the hierarchical mixing angles $\sin \theta_{\psi}$ can be naturally generated in the warped extra dimension picture.

The mixing angles for gauge bosons are given by $\sin \theta_{G}=\frac{g_{e l}}{g_{*}}$, where $g_{e l}$ is the elementary gauge coupling, while the SM gauge coupling is given by $g_{S M}=g_{e l} g_{*} / \sqrt{g_{e l}^{2}+g_{*}^{2}}$. We will choose $g_{*} \sim$ a few such that $g_{*} \gg g_{S M}$ and thus $g_{e l}$ can be approximated by the SM gauge couplings. Specifically, in order to match $5 \mathrm{D}$ theories $\sin \theta_{G}$ should be $\sim 1 / \sqrt{\text { logarithm of UV-IR hierarchy }}$, i.e., $\sim 1 / 6$ for the case of Planck-weak hierarchy. Here we review the couplings of heavy gauge bosons $\rho_{\mu}$ to SM states, which we use to compare with the couplings of coset gauge bosons later. The SM fermion coupling to heavy gauge bosons are generated both through fermionic and gauge boson mixings. This is illustrated using insertion approximation in Fig. 1 (A)(B) . This gives the coupling

$$
g_{\rho \psi \psi} \approx \sin \theta_{\psi} g_{*} \sin \theta_{\psi}+\frac{g_{e l}^{2}}{g_{*}} .
$$

Note that there is a flavor dependent contribution (first term in the above equation) that comes from elementary composite mixing of fermions, which is suppressed by the fermionic mixing angles $\sin \theta_{\psi_{L, R}}$, and there is a flavor universal contribution (second term in the above equation) that comes

\footnotetext{
${ }^{9}$ The SM states and heavy eigenstates further mix with each other after EWSB, but this effect is not relevant here.
} 


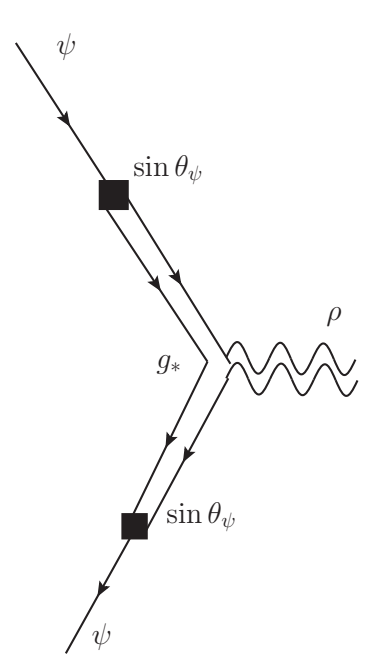

$(A)$

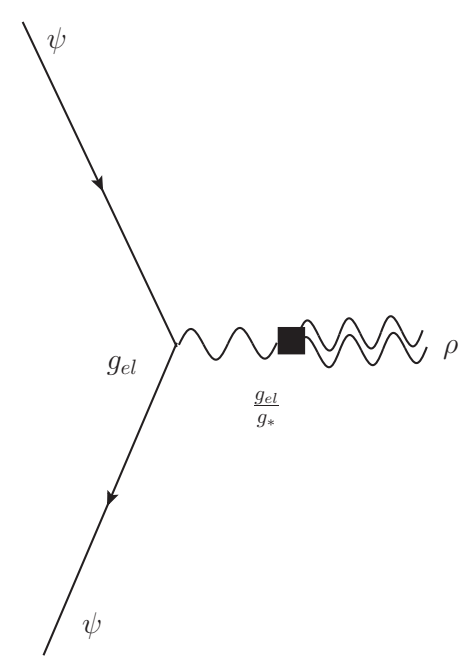

$(B)$

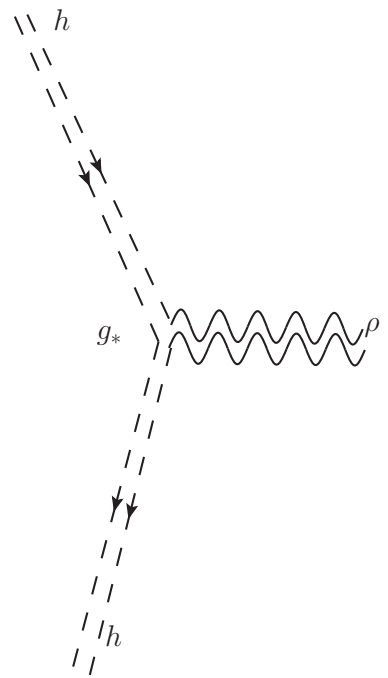

$(C)$

Figure 1: Couplings of heavy gauge bosons with SM states. Fig. (A), (B) give the couplings between heavy gauge bosons and SM fermions coming from fermionic and gauge boson mixings, respectively. Fig. (C) gives the coupling between heavy gauge bosons and Higgs field, which after EWSB give rise to the coupling to physical Higgs and longitudinal $W / Z$.

from elementary/composite mixing of gauge bosons, which is suppressed by $\frac{g_{e l}}{g_{*}}$ relative to SM gauge couplings. For light fermions, the flavor universal term dominates. Moreover, this coupling is only mildly (i.e., $\sim 1 / 6$ ) suppressed relative to the SM one. The heavy gauge bosons couple strongly to Higgs field since they are both mostly composite states (See Fig. 1(C)):

$$
g_{\rho h h} \approx g_{*} .
$$

Using Goldstone boson equivalence theorem, we can see that after EWSB, the heavy gauge bosons acquire strong coupling $\approx g_{*}$ with physical Higgs boson and longitudinal component of $W / Z$.

We now turn to the two-site description of warped/composite $P G B$ Higgs. First let us ignore $S U(3)_{c} \times U(1)_{X}$ part of the composite sector global symmetry because it is irrelevant for the Higgs part of the model. We want composite sector of the model to have a global symmetry $H$ which includes $S U(2)_{L} \times S U(2)_{R}$ [latter group is isomorphic to $\mathrm{SO}(4)$ ]. At the same time, Higgs should be a PGB. One can see that in order to achieve this setup, the composite sector should have larger global symmetry $G$, which later should be spontaneously broken down to its subgroup $H$, and Higgs is PGB of this symmetry breaking pattern $G \rightarrow H$ in the composite sector. The simplest example which we will study in this paper corresponds to the $G=S O(5)$ and $H=S O(4)$, i.e., the full global symmetry of the composite sector is extended from $S U(3)_{c} \times S U(2)_{L} \times S U(2)_{R} \times U(1)_{X}$ of the original model to $S U(3)_{c} \times S O(5) \times U(1)_{X}$. One can see that due to the larger symmetry $G$ of 
the composite sector there will be additional heavy gauge bosons which belong to the group $G / H$ (i.e., the coset), and they correspond to the coset gauge bosons of the general warped/composite PGB Higgs framework.

We can learn some important properties of the coset gauge bosons based on this simple setup. First, we argue that coset gauge bosons are generally heavier than the usual composite gauge bosons (i.e. the gauge bosons of the gauge group $H$ ). The argument is the following. Before the symmetry breaking $G \rightarrow H$, the gauge bosons of $H\left(\rho^{\mu}\right)$ and $G / H\left(\rho_{c}^{\mu}\right)$ of the composite sector should have the same mass (due to the global symmetry, $G$ ). After the symmetry breaking, the masses of the gauge bosons of $H$ remain the same, while the coset gauge bosons in $G / H$ get extra mass contribution coming from the breaking. For example, for the case that we consider, i.e., with $G=S O(5)$ and $H=S O(4)$, the breaking $G \rightarrow H$ can be achieved by the vev of a scalar $\phi$ transforming in fundamental representation of $S O(5)$. We can parameterize $\phi$ by

$$
\phi=e^{-i T_{c}^{a} h^{a}}\left(\begin{array}{c}
0 \\
0 \\
0 \\
0 \\
f_{\phi}+\eta
\end{array}\right)
$$

where $f_{\phi}$ is the magnitude of $\phi$ vev, $T_{c}^{a}$ are the generators of $G / H, h^{a}$ are the pseudo-Goldstone bosons which are also the Higgs, $\eta$ is a massive scalar excitation. The covariant derivative of $\phi$ gives rise to extra contribution to the masses of coset gauge bosons

$$
\left(D_{\mu} \phi\right)^{\dagger}\left(D^{\mu} \phi\right) \supset g_{*}^{2} f_{\phi}^{2} \rho_{c, \mu} \rho_{c}^{\mu}
$$

This extra contribution is always positive, thus $\rho_{c}^{\mu}$ are generally heavier than $\rho^{\mu} 10$. We conclude that

- there is an indirect bound of $\gtrsim 3 \mathrm{TeV}$ for the coset gauge boson masses, which comes from the bound (from precision tests) of $\sim 3 \mathrm{TeV}$ on the ordinary composite gauge bosons (as mentioned earlier).

On the other hand, since it is the coset gauge bosons that cancel the quadratic divergence in Higgs mass from $W / Z$ loops, it is clear that naturalness favors the coset gauge bosons to not be heavier than several $\mathrm{TeV}$. We can also study the structure of coset gauge boson couplings based on the two-site language. Note that the discussion here is independent of the scale $f_{\phi}$ (see Eq. (6) ) that controls the masses of coset gauge bosons (relative to the other gauge bosons)11. We will see that the quantum numbers of coset gauge bosons give important restrictions on their couplings. First,

\footnotetext{
${ }^{10}$ assuming only the minimal couplings of $\phi$ to coset gauge bosons as above.

${ }^{11} f_{\phi}$ is also (roughly) related to the size of the scalar vev breaking $S O(5) \rightarrow S O(4)$ in the $5 \mathrm{D}$ model.
} 
we study their couplings with two SM gauge bosons. For this purpose, we consider the SM gauge bosons before EWSB: $W_{\mu}^{a}$ transform in adjoint representation of $S U(2)_{L}$ and $B_{\mu}$ transform as a singlet. The SM quantum numbers of coset gauge bosons are the same as that of Higgs, i.e., they are $S U(2)_{L}$ doublet. Just based on quantum numbers, we can see that

- there is no coupling between one coset gauge boson and two SM gauge bosons or two Higgs bosons at lowest order (i.e., without EWSB),

which is independent of the elementary/composite nature of SM/coset gauge bosons. This is to be contrasted with Eq. (4) and Fig. 1(C), where we see that the usual heavy gauge bosons have large couplings to Higgs bosons and longitudinal $W / Z$.

We turn to the couplings between coset gauge bosons and SM fermions. We denote the SM fermions by $q_{L}\left(S U(2)_{L}\right.$ doublet) and $u_{R}\left(S U(2)_{L}\right.$ singlet) respectively, where $L, R$ subscripts stand for the $4 \mathrm{D}$ chirality 12 Based on quantum numbers, we cannot write down dimension 4 coupling between SM fermions and coset gauge bosons. The only allowed dimension 4 couplings are

$$
g_{q U} \bar{q}_{L} \gamma_{\mu} \rho_{c}^{\mu} U_{L}+g_{u Q} \bar{u}_{R} \gamma_{\mu} \rho_{c}^{\mu} Q_{R}+\text { h.c. }
$$

where $Q_{R}, U_{L}$ are heavy (purely composite) fermionic states transforming under $S U(2)_{L}$ as doublet and singlet, respectively, i.e., opposite chirality to the SM fermions. Recall that the composite sector fermions are in vector-like and complete representations of $S O(5)$ (in particular, the SM gauge group), while the elementary sector fermions are only in complete, chiral representation of SM gauge group.

There could be higher dimensional operators that couple coset gauge bosons with just SM fermions. These couplings can be schematically written as:

$$
\frac{\tilde{g}_{q}}{\Lambda} \bar{q}_{L} \gamma_{\mu} \rho_{c}^{\mu} h q_{L}+\frac{\tilde{g}_{u}}{\Lambda} \bar{u}_{R} \gamma_{\mu} \rho_{c}^{\mu} h u_{R}
$$

where $\Lambda$ is some mass scale which depends on the specific model. There could also be magnetic dipole moment type operators involving just SM fermions and coset gauge bosons:

$$
\frac{g_{\text {dipole }}}{\Lambda} \bar{q}_{L} \sigma^{\mu \nu} D_{[\mu} \rho_{\nu]}^{c} u_{R}+\text { h.c. },
$$

where $D_{\mu}$ is the covariant derivative operator with respect to $S U(2)_{L} \times U(1)_{Y}$.

So far we have been analyzing the couplings of the coset gauge bosons based only on their quantum numbers without implementing any specific property of the model. Let us now first estimate the size of the couplings $g_{q U, u Q}$ in Eq. (77) based on our two-site description of the general

\footnotetext{
${ }^{12}$ Couplings of coset gauge bosons to right-handed down-type quarks and leptons can be similarly studied, but these states are not relevant here since the associated elementary-composite mixings (even for bottom quark and $\tau$, i.e., the heaviest fermions) are small and, as we will discuss later, these sectors also do not result in light KK states.
} 


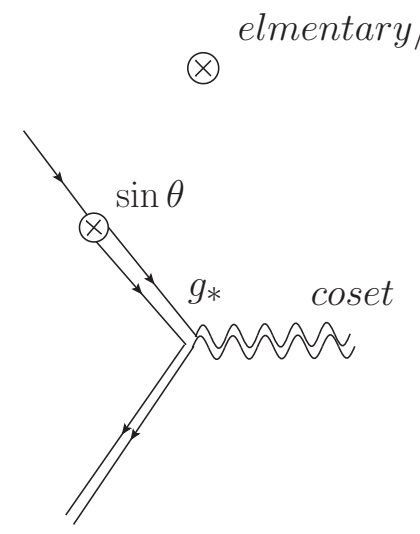

(A)

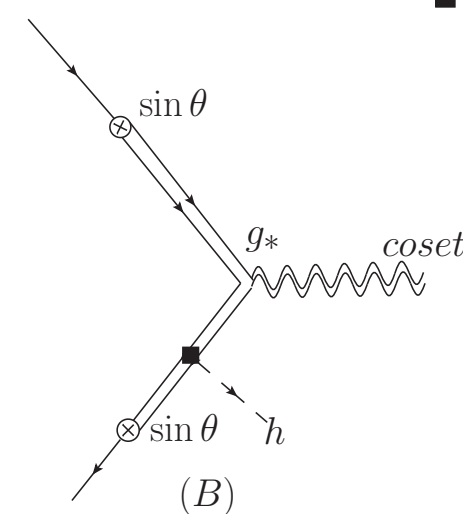

$(B)$
Higgs insertion

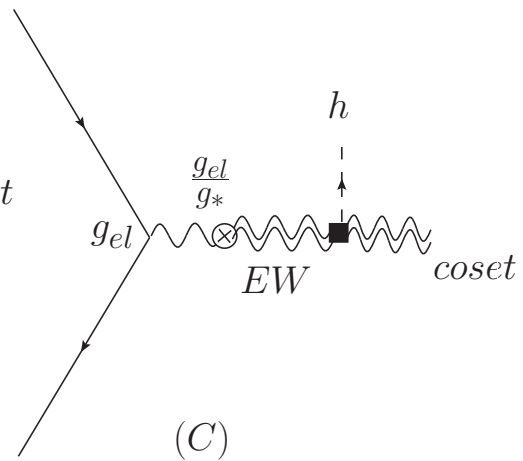

Figure 2: Couplings between coset gauge bosons and fermions using insertion approximation. Fig. (A) shows the couplings between coset gauge boson, SM fermion and composite fermion. Estimates of these couplings are given in Eq. (10). Fig. (B) shows the couplings between coset gauge boson and two SM fermions coming from elementary/composite mixing of fermions. A Higgs insertion is needed since otherwise the composite fermion cannot mix with elementary fermion due to quantum numbers, namely, this composite fermion has opposite-to-SM chirality. Estimates of these couplings are given in Eq. (11). Fig. (C) shows the couplings between coset gauge boson and two SM fermions coming from the mixing of elementary and composite gauge bosons of the SM-type (denoted by "EW") followed by their mixing with coset gauge bosons induced by the Higgs vev. Estimates of these couplings are given in Eq. (12).

warped/composite PGB Higgs framework, i.e., utilizing the elementary or composite sector nature of the various particles. Since the SM fermions are mostly elementary, the above couplings must arise due to elementary/composite fermionic mixing. In the insertion approximation the couplings

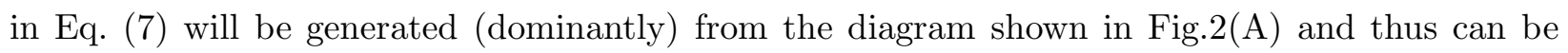
estimated to be:

$$
g_{q U} \sim g_{*} \sin \theta_{q_{L}}, \quad g_{u Q} \sim g_{*} \sin \theta_{u_{R}}
$$

For the third generation quarks (especially top quark), it is possible that $\sin \theta_{t_{L, R}}, \sin \theta_{b_{L}} \sim O(1)$. Therefore,

- coset gauge boson should couple strongly with $t_{L, R}, b_{L}$ and composite fermions.

Turning now to the couplings in Eq. (8), they can be generated via elementary-composite fermion mixing, as shown in Fig,2(B) in insertion approximation. Thus the mass scale $\Lambda$ of Eq. (8) in the two-site description of PGB Higgs becomes equal to the mass of composite sector fermions (denoted by $M_{*}$ ) and the order of the coupling factor $\tilde{g}$ can be estimated to be $g_{*} \sin \theta_{\psi}^{2}$. Once Higgs gets vev, the couplings between coset gauge bosons and SM fermions can then be generated:

$$
g_{q q}^{f . d .} \sim \frac{v}{M_{*}} g_{*}^{2}\left(\sin \theta_{q_{L}}\right)^{2}, \quad g_{u u}^{f . d .} \sim \frac{v}{M_{*}} g_{*}^{2}\left(\sin \theta_{u_{R}}\right)^{2},
$$


where "f.d." denotes flavor-dependent couplings.

Another contribution to these couplings comes from elementary-composite $S M$-type gauge boson mixing - recall that there is no elementary coset gauge boson, followed by composite SM-coset mixing via Higgs vev, as shown in Fig. 2(C) with

$$
g_{q q, u u}^{f . i .} \sim \frac{g_{e l}^{2}}{g_{*}} \frac{g_{*} v}{M_{*}}
$$

Clearly, these couplings are flavor-independent (hence denoted by "f.i.") and dominate the ones in Eq. (11) for light SM fermions, whereas those in Eq. (11) dominate for third generation quarks. The magnetic dipole moment type operator (Eq. (9)) is recently discussed in [18. In our framework, this operator is only generated through loop processes, and it is further suppressed by the fermion mixing angle $\left(\sin \theta_{\psi}\right)^{2}$. Therefore, it is not phenomenologically important here, even for top/bottom quarks.

We can now study the phenomenological implications of these couplings of coset gauge bosons. As studied in [30], the dominant production channel for the "usual" (i.e., transforming as adjoint of SM gauge group) heavy gauge bosons is through the (flavor-universal) coupling between light quarks and heavy gauge bosons (see second term of Eq. (3) and Fig. 1(B)). However, as argued above, the coupling of coset gauge bosons to two light quarks is suppressed by $\sim g_{*} v / M_{*}$ compared to similar couplings of usual heavy gauge bosons. Since for realistic models one usually finds $g_{*} v / M_{*} \lesssim 0.4$, the resonant production of coset gauge bosons via light quarks is expected to be suppressed by at least an order of magnitude compared to that of usual heavy gauge bosons (for the same mass).

On the other hand, the dominant discovery channel for usual heavy gauge boson is via decay into two Higgs or two (longitudinal) W/Z gauge bosons or into two third generation quarks due to the composite sector nature of all these particles. However, the main decay channels for coset gauge bosons are into one third generation SM quark and one heavy quark based on above analysis; of course, for this decay to be kinematically allowed, the heavy quark must be lighter than coset gauge bosons - we find that such a scenario does indeed occur in part of the parameter space 13 . Again, even if $\sin \theta_{t_{L, R}, b_{L}} \sim O(1)$, the decay into two third generation SM quarks is suppressed compared to the decay into one third generation quark and one heavy quark due to suppression of the former coupling by $\sim g_{*} v / M_{*}$ relative to the latter. And, couplings of coset gauge bosons to light quarks are even smaller.

We can see that the phenomenology of coset gauge bosons is very distinct from that of usual heavy gauge bosons so that the two types of gauge bosons can be distinguished based on their signals at the LHC. Note that the conclusion here is general in the sense that it is the result of the

\footnotetext{
${ }^{13}$ The coupling of coset gauge bosons to two heavy (mostly composite) fermions is also large, but we find that (typically) such a decay is not kinematically possible and hence this coupling is not relevant for our analysis.
} 
quantum numbers of coset gauge bosons and their (purely) composite sector nature. This renders our collider study to be robust and not dependent on specific models.

\section{1 "Pollution" from the usual heavy gauge bosons in the signal from the resonant production of coset gauge bosons}

Finally, we would like to emphasize that a study of channels other than resonant production (via light quarks) for coset gauge bosons is motivated for the following reasons. The point is that for resonant production of coset gauge bosons, even though the coset gauge bosons have distinctive decays (as discussed above), it turns out in the end that there is a larger contribution from resonant production of the usual heavy gauge bosons (i.e., composite sector $W / Z$ 's) to the same final states. Given that coset gauge boson is a doublet of $S U(2)_{L}$, it is clear that the dominant fermionic decay (i.e., not requiring EWSB) of coset gauge bosons is to a doublet and singlet (whether SM or heavy) - we will focus here on final state with SM top/bottom and composite (heavy, i.e., non-SM) fermion. Whereas, the usual heavy gauge bosons are triplets/singlets and so cannot decay without EWSB into this final fermionic state, but instead decay into two doublets or two singlets. However, after EWSB, the usual heavy gauge bosons can decay into the same final state as the coset gauge boson. One possibility is EWSB mixing on gauge boson line, i.e., the usual heavy gauge bosons do have an admixture of coset gauge bosons [again, resulting from the Higgs vev as shown in Fig. 2(C)]. Hence, via this coset gauge boson component, the usual heavy gauge bosons will decay into the same final state as that of the coset. Of course, this effect does not really constitute a "pollution" since it does require presence of the coset gauge boson, i.e., in this case, the top/bottom and composite fermion final state can still be taken as "evidence" for coset gauge boson.

However, another possibility is EWSB mixing on fermion line: the composite sector $W / Z$ 's decay into two doublets or two singlet fermions, followed by doublet mixing with a singlet (or vice versa) via EWSB, i.e., the fermionic mass eigenstates are also admixtures of doublet and singlet. The crucial point is that this decay of usual heavy gauge boson to the same final state as that of coset gauge boson can occur even in the absence of the coset gauge boson and hence is a genuine pollution. Of course, such decays of usual heavy gauge bosons will be suppressed by this EWSB mixing, i.e., factors of $g_{*} v / M_{*}$ (or $Y_{*} v / M_{*}$ ), compared to other final states such as $W W / W Z$ and $t \bar{t} / t \bar{b}$ to which the usual heavy gauge bosons couple strongly. However, it is clear that the above suppression in the decay of usual heavy gauge bosons to the same final state as for coset gauge boson simply serves to compensate (in the total amplitude) the larger coupling (as mentioned above) of the usual heavy gauge bosons to the initial state light quarks. Moreover, given that the coset gauge bosons are heavier than usual gauge bosons (they cannot be lighter as suggested by the twosite description), the PDF's will then result in the contribution to the top/bottom and composite 
fermion final state from the production/decay of usual heavy gauge bosons actually dominating that from coset gauge bosons.

Thus, in this case, the pollution from usual heavy gauge bosons might make it difficult to extract a signal for the coset gauge bosons from their resonant production via light quark annihilation. Of course, we could undertake the difficult task of reconstruction of the invariant mass of the final state of top/bottom and composite fermion in order to separate the two contributions (again, coset gauge bosons are generically heavier than the usual heavy gauge bosons). Thus, a very careful study (i.e., including production of usual heavy gauge bosons), which is beyond the scope of this paper, would be required to ascertain whether resonant production via light quarks is actually a useful channel. Therefore, in section 5, we will pursue another channel (namely associated production of $W_{C}$ ) which has comparable cross-section to resonant production via light quarks and furthermore has no significant pollution from production of the usual heavy gauge bosons.

\section{Gauge-Higgs Unification in Warped Extra Dimension}

\subsection{Gauge Bosons and Higgs Fields}

Having discussed general two-site description of the general warped/composite PGB Higgs framework, we now turn to a specific 5D model. In this section, we review models of (minimal) GHU in a warped extra dimension: for more details, see reference [8] whose notation we will mostly follow here (see also references [16, 21] for similar analyses). The spacetime metric is given by [1]

$$
d s^{2}=\frac{1}{(k z)^{2}}\left\{\eta_{\mu \nu} d x^{\mu} d x^{\nu}-d z^{2}\right\}, \quad z \in\left[R, R^{\prime}\right]
$$

where $k$ is the curvature scale, $R=\frac{1}{k}, R^{\prime}=\frac{e^{k L}}{k}$, and $L$ is the (proper) length of the fifth dimension which we choose to be $\sim \frac{35}{k}$ to explain the Planck-weak hierarchy. The Standard Model (SM) gauge group $S U(3)_{C} \times S U(2)_{L} \times U(1)_{Y}$ is a subgroup of the bulk gauge symmetry. To be specific, we take the bulk gauge symmetry to be $S U(3)_{C} \times S O(5) \times U(1)_{X}$ in the following analysis (the group algebra of $S O(5)$ can be found in Appendix $\mathrm{A}$ ). We will drop the color group $S U(3)_{C}$ in the following analysis since it does not affect our result. The gauge boson action is given by

$$
S_{g}=\int d^{5} x \sqrt{-G}\left[-\frac{1}{2 g_{5}^{2}} \operatorname{Tr}\left(F^{(A) M N} F_{M N}^{(A)}\right)-\frac{1}{4 g_{X}^{2}} F^{(X) M N} F_{M N}^{(X)}\right],
$$

with

$$
A_{M}=\sum_{a=1}^{3} A_{M}^{a_{L}} T_{L}^{a}+\sum_{a=1}^{3} A_{M}^{a_{R}} T_{R}^{a}+\sum_{\hat{a}=1}^{3} A_{M}^{\hat{a}} T^{\hat{a}}+A_{M}^{\hat{4}} T^{\hat{4}}
$$

where $T_{L, R}^{a}$ are the generators of $S U(2)_{L} \times S U(2)_{R} \cong S O(4) \subset S O(5)$, and $T^{\hat{a}, \hat{4}}$ are the generators of the coset $S O(5) / S O(4) . X_{M}$ is the gauge boson of $U(1)_{X}$. The boundary conditions are chosen such 
that only the subgroup $S U(2)_{L} \times U(1)_{Y}$ is unbroken at UV brane $(z=R)$ and $S U(2)_{L} \times S U(2)_{R} \times$ $U(1)_{X} \cong S O(4) \times U(1)_{X}$ is unbroken at IR brane $\left(z=R^{\prime}\right)$, where the hypercharge $\mathrm{Y}$ is defined as $\frac{Y}{2}=T_{R}^{3}+Q_{X}$. Specifically, we choose the $A_{\mu}(\mu=0,1,2,3)$ components of $S U(2)_{L} \times U(1)_{Y}$ and $S O(4) \times U(1)_{Y}$ to have Neumann boundary condition ("+") on the UV brane and IR brane respectively, and all the other $A_{\mu}(\mu=0,1,2,3)$ have Dirichlet boundary condition ("-_") on both branes. To reproduce hypercharge in Standard Model, we do the following rotation of fields [8]

$$
\begin{gathered}
\left(\begin{array}{c}
A_{M}^{3_{R}} \\
B_{M}^{Y}
\end{array}\right)=\left(\begin{array}{cc}
c_{\phi} & -s_{\phi} \\
s_{\phi} & c_{\phi}
\end{array}\right)\left(\begin{array}{c}
A_{M}^{3_{R}} \\
X_{M}
\end{array}\right), \\
c_{\phi}=\frac{g_{5}}{\sqrt{g_{5}^{2}+g_{X}^{2}}}, \quad s_{\phi}=\frac{g_{X}}{\sqrt{g_{5}^{2}+g_{X}^{2}}},
\end{gathered}
$$

where we need $s_{\phi}^{2} \approx \tan ^{2} \theta_{W} \approx 0.30$ to get the correct Weinberg angle. Based on this definition, we set $B_{\mu}^{Y}$ to have "+" boundary condition on both branes, and $A^{\prime 3_{R}}$ to have "-" boundary condition on UV brane and "+" boundary condition on IR brane. With this set of assignment of boundary conditions, we can reproduce SM gauge group at low energy, while at the same time preserve $S U(2)_{L} \times S U(2)_{R}$ custodial symmetry [4].

An important observation here is that for gauge fields $A_{M}$, its $A_{\mu}$ and $A_{z}$ components should have

opposite boundary conditions on the branes. This means that $A_{z}^{\hat{a}, \hat{4}}$ have "+" boundary conditions on both branes, thus there are zero modes associated with them. We identify these zero modes of $A_{z}^{\hat{a}, \hat{4}}$ as the Higgs fields $H^{a, 4}$. They transform as a doublet under $S U(2)_{L}$, thus have the same gauge quantum numbers of SM Higgs. Due to 5D gauge invariance, these Higgs fields are massless at tree level, and their potential is generated by the breaking of $S O(5)$ on UV and IR branes. Therefore, the Higgs potential will be generated through loop effects. Since from 5D point of view, this is a non-local effect, the generated Higgs potential will be finite. We will discuss the mechanism of radiative generation of Higgs potential later in this section.

\subsection{Fermions}

The fermions also propagate in the bulk, with the following action

$$
S_{f}=\int d^{5} x \sqrt{-G} \sum_{i} \bar{\Psi}_{i}\left(i \Gamma^{M} D_{M}-c_{i} k\right) \Psi_{i}
$$

where $D_{M}=\partial_{M}-i A_{M}-i X_{M}$ and $c_{i}$ are the bulk masses of the $5 \mathrm{D}$ fermions in units of $k$, which control the localization of fermion zero modes. To be specific, the zero modes for left-handed (right-handed) fermions are localized near UV brane if $c>1 / 2(c<-1 / 2)$, and they are localized near IR brane if $c<1 / 2(c>-1 / 2)$. For future use, we define

$$
f(c)=\sqrt{\frac{1 / 2-c}{1-e^{-(1-2 c) k L}}},
$$


which is the size of zero mode fermion wavefunction at IR brane in units of $\sqrt{2 k}$. There are various scenarios to embed SM fermions into representations of $S O(5)[\underline{8}, 11,21$. For the following discussion, we just consider the third generation fermions, since the first two generation fermions are not important for EWSB and collider phenomenology. For concreteness, we follow [8] and choose the fermion representation to be $5 \oplus 5 \oplus 10$ for one generation. The generators of $S O(5)$ for 5 representation can be found in Appendix $\mathrm{A}$. The fermions in $\mathbf{5}$ of $S O(5)$ have the following charge assignment under $S U(2)_{L} \times S U(2)_{R}$ :

$$
\mathbf{5}=\frac{1}{\sqrt{2}}\left(\begin{array}{c}
i q_{++}+i q_{--} \\
q_{--}-q_{++} \\
i q_{-+}-i q_{+-} \\
q_{-+}+q_{+-} \\
\sqrt{2} q^{c}
\end{array}\right)
$$

where \pm means $\pm 1 / 2$ under $S U(2)_{L}$ and $S U(2)_{R}$ respectively, and $q^{c}$ means singlet. A more convenient basis is

$$
\xi_{5}=\left(\begin{array}{c}
q_{++} \\
q_{-+} \\
q_{+-} \\
q_{--} \\
q^{c}
\end{array}\right) \equiv\left(\begin{array}{c}
\chi \\
\tilde{t} \\
t \\
b \\
\hat{t}
\end{array}\right)
$$

where $\chi, t, b$ denote fermions with charge $+5 / 3,+2 / 3,-1 / 3$ respectively. The transformation between the two basis is

$$
\xi_{5}=A \times \mathbf{5} \quad \text { with } A=\frac{1}{\sqrt{2}}\left(\begin{array}{ccccc}
-i & -1 & 0 & 0 & 0 \\
0 & 0 & -i & 1 & 0 \\
0 & 0 & i & 1 & 0 \\
-i & 1 & 0 & 0 & 0 \\
0 & 0 & 0 & 0 & \sqrt{2}
\end{array}\right)
$$

The fermions are embedded in $\mathbf{1 0}$ of $S O(5)$ as follows

$$
\mathbf{1 0}=\left(\begin{array}{cccccccccc}
\chi & \tilde{t} & t & b & \Xi^{\prime} & T^{\prime} & B^{\prime} & \Xi & T & B
\end{array}\right)^{T}
$$

where $\left(\begin{array}{cc}\chi & t \\ \tilde{t} & b\end{array}\right)$ form an $S U(2)_{L} \times S U(2)_{R}$ bidoublet, $(\Xi, T, B)$ form $S U(2)_{R}$ triplet, and $\left(\Xi^{\prime}, T^{\prime}, B^{\prime}\right)$ form $S U(2)_{L}$ triplet. We can also write down the 10 representation in the form of $5 \times 5$ matrix

$$
\xi_{10}=\frac{1}{2}\left(\begin{array}{ccccc}
0 & T^{\prime}+T & i \frac{B^{\prime}-\Xi^{\prime}}{\sqrt{2}}+i \frac{B-\Xi}{\sqrt{2}} & \frac{B^{\prime}+\Xi^{\prime}}{\sqrt{2}}-\frac{B+\Xi}{\sqrt{2}} & b+\chi \\
-T^{\prime}-T & 0 & \frac{B^{\prime}+\Xi^{\prime}}{\sqrt{2}}+\frac{B+\Xi}{\sqrt{2}} & -i \frac{B^{\prime}-\Xi^{\prime}}{\sqrt{2}}+i \frac{B-\Xi}{\sqrt{2}} & i(b-\chi) \\
-i \frac{B^{\prime}-\Xi^{\prime}}{\sqrt{2}}-i \frac{B-\Xi}{\sqrt{2}} & -\frac{B^{\prime}+\Xi^{\prime}}{\sqrt{2}}-\frac{B+\Xi}{\sqrt{2}} & 0 & T^{\prime}-T & t+\tilde{t} \\
-\frac{B^{\prime}+\Xi^{\prime}}{\sqrt{2}}+\frac{B+\Xi}{\sqrt{2}} & i \frac{B^{\prime}-\Xi^{\prime}}{\sqrt{2}}-i \frac{B-\Xi}{\sqrt{2}} & -T^{\prime}+T & 0 & -i(t-\tilde{t}) \\
-b-\chi & -i(b-\chi) & -t-\tilde{t} & i(t-\tilde{t}) & 0
\end{array}\right) \cdot(24
$$


The fermion content and the boundary condition assignment can be summarized as follows

$$
\Psi_{1 L}=\left(\begin{array}{c}
\chi_{1 L}(-,+) \\
\tilde{t}_{1 L}(-,+) \\
t_{1 L}(+,+) \\
b_{1 L}(+,+) \\
\hat{t}_{1 L}(-,+)
\end{array}\right), \quad \Psi_{2 R}=\left(\begin{array}{c}
\chi_{2 R}(-,+) \\
\tilde{t}_{2 R}(-,+) \\
t_{2 R}(-,+) \\
b_{2 R}(-,+) \\
\hat{t}_{2 R}(+,+)
\end{array}\right), \quad \Psi_{3 R}=\left(\begin{array}{c}
\chi_{3 R}(-,+) \\
\tilde{t}_{3 R}(-,+) \\
t_{3 R}(-,+) \\
b_{3 R}(-,+) \\
\Xi_{3 R}^{\prime}(-,+) \\
T_{3 R}^{\prime}(-,+) \\
B_{3 R}^{\prime}(-,+) \\
\Xi_{3 R}(-,+) \\
T_{3 R}(-,+) \\
B_{3 R}(+,+)
\end{array}\right),
$$

while the opposite chirality fields have the opposite boundary conditions. From this set of boundary conditions, we can see that there are fermion zero modes for one $S U(2)_{L}$ doublet and two $S U(2)_{L}$ singlets, which reproduce the SM fermion gauge representations at low energy. To get SM fermion masses, we need the following boundary mass terms

$$
S_{b}=\int d^{5} x \sqrt{-G} 2(k z) \delta\left(z-R^{\prime}\right)\left[M_{B_{1}} \overline{\hat{t}}_{1 L} \hat{t}_{2 R}+M_{B_{2}}\left(\bar{\chi}_{1 L}, \overline{\tilde{t}}_{1 L}, \bar{t}_{1 L}, \bar{b}_{1 L}\right)\left(\begin{array}{c}
\chi_{3 R} \\
\tilde{t}_{3 R} \\
t_{3 R} \\
b_{3 R}
\end{array}\right)+\text { h.c. }\right] .
$$

We have to choose the parameters $c_{1}, c_{2}, c_{3}, M_{B_{1}}, M_{B_{2}}$ to reproduce the top and bottom masses.

\subsection{Higgs Potential and KK Decomposition}

We have identified Higgs fields as the 5th components of the gauge fields of coset $S O(5) / S O(4)$. Here, we briefly review the KK decomposition of bulk fields with a background Higgs fields and how the potential of Higgs is radiatively generated . For more details, see [8, 31].

We denote $A_{\mu}^{a}$ as the gauge bosons of $S U(2)_{L} \times S U(2)_{R}$ and $A_{\mu}^{\hat{a}}(\hat{a}=1 \ldots 4)$ as the gauge bosons of $S O(5) / S O(4)$. The zero mode of $A_{z}^{\hat{a}}$ gives the Higgs. We can do the following KK decomposition

$$
\begin{aligned}
A_{\mu}^{a}(x, z) & =\sum_{n} f_{n}^{a}(z, v) A_{\mu, n}(x), \\
A_{5}^{a}(x, z) & =\sum_{n} \frac{\partial_{z} f_{n}^{a}(z, v)}{m_{n}(v)} h_{n}(x), \\
A_{\mu}^{\hat{a}}(x, z) & =\sum_{n} f_{n}^{\hat{a}}(z, v) A_{\mu, n}(x), \\
A_{5}^{\hat{a}}(x, z) & =C_{h} h^{\hat{a}}(x) k z+\sum_{n} \frac{\partial_{z} f_{n}^{\hat{a}}(z, v)}{m_{n}(v)} h_{n}(x) .
\end{aligned}
$$

We need $C_{h}=\sqrt{\frac{2 k}{\left(e^{2 k L}-1\right)}} g_{5}$ to make the Higgs field canonically normalized. Note that all the wavefunctions depend on the vev of Higgs $\left(\left\langle h^{\hat{4}}\right\rangle=v\right)$. The boundary conditions for these wave- 
functions are complicated. However, the wavefunctions with non-vanishing Higgs vev are related to the wavefunctions with vanishing Higgs vev by a gauge transformation 31]

$$
f^{\alpha}(z, v) T^{\alpha}=\Omega^{-1}(z, v) f^{\alpha}(z, 0) T^{\alpha} \Omega(z, v)
$$

with

$$
\Omega(z, v)=e^{-i C_{h} v T^{\hat{4}} \int_{R}^{z} d z^{\prime} k z^{\prime}}=\exp \left[-i C_{h} v T^{\hat{4}} k\left(z^{2}-R^{2}\right) / 2\right] \equiv \exp \left[-i \frac{v\left(z^{2}-R^{2}\right)}{f_{h}\left(R^{\prime 2}-R^{2}\right)} T^{\hat{4}}\right]
$$

where we defined the "Higgs decay constant" $f_{h} \equiv \frac{\sqrt{2 k}}{g_{5} \sqrt{e^{2 k L}-1}}$. Therefore, to simplify the task, we can just calculate the wavefunctions with vanishing Higgs vev, and do a transformation $\Omega(z, v)$ to find the wavefunctions with non-vanishing Higgs vev. We then apply boundary conditions for the wavefunctions $f^{a, \hat{a}}(z, v)$ on the IR brane to get the mass spectrum of gauge KK modes. The details of the calculation are shown in Appendix B. In the end, we get two spectral functions $\rho_{W, Z}(m, v)$ for $W, Z$ bosons (Eqs. (105) and (115)), whose roots give us the mass spectra $m_{W, Z}^{n}$ for $W, Z$ bosons. Similarly, the wavefunctions for fermions with non-vanishing Higgs vev $F_{1,2,3}^{\Psi}(z, v)$ are also related to the wavefunctions for fermions with vanishing Higgs vev $F_{1,2,3}^{\Psi}(z, 0)$ by the gauge transformation $\Omega(z, v)$ :

$$
\begin{aligned}
F_{1,2}^{\Psi}(z, v) & =A \Omega(z, v)^{-1} A^{-1} F_{1,2}^{\Psi}(z, 0) \\
F_{3}^{\Psi}(z, v) & =\Omega(z, v)^{-1} F_{3}^{\Psi}(z, 0) \Omega(z, v)
\end{aligned}
$$

where we have written $F_{1,2}^{\Psi}$ in the basis specified in Eq. (21) and $F_{3}^{\Psi}$ in the form of $5 \times 5$ matrix (see Eq. (24)), and matrix $A$ is defined in Eq. (22). Similarly to the gauge boson case, we can get spectral functions for top and bottom quarks $\rho_{t, b}(m, v)$ (Eqs. (131) and (132)), whose roots give us the mass spectra $m_{t, b}^{n}$ for $t, b$ fermions. We can calculate the Coleman-Weinberg potential for Higgs once we know all the spectral functions 31]

$$
V_{C W}^{W}(v)=\frac{1}{(4 \pi)^{2}} \int_{0}^{\infty} d p p^{3}\left\{6 \ln \left[\rho_{W}(i p, v)\right]+3 \ln \left[\rho_{Z}(i p, v)\right]-12 \ln \left[\rho_{t}(i p, v)\right]-12 \ln \left[\rho_{b}(i p, v)\right]\right\} .
$$

This integral can be done numerically. We can minimize this potential to find the Higgs vev $v$. Then we can find the mass spectra of the model through the spectral functions $\rho_{W, Z, t, b}(m, v)$.

\subsection{Couplings of Coset Gauge Bosons}

\subsubsection{Estimates and General Patterns}

The exact couplings for coset gauge bosons involve overlap integrals of wavefunctions, which has to be done numerically and hence are not very illuminating. We defer showing the formulae for exact couplings to section 4.4 .2 and a discussion of the numerical analysis to section 4.5. In order to gain 
some insights into the structure of coset gauge boson couplings, we concentrate here on estimating the sizes of the couplings between both charged $\left(W_{C}\right)$ and neutral $\left(Z_{C}\right)$ coset gauge bosons and fermions based on 5D profiles, and we will show that the results here match the ones coming from two-site description shown earlier in section 314 In the following analysis, we focus on the parametric dependence of these couplings on $\theta_{H} \equiv \frac{h}{\sqrt{2} f_{h}}$ and wavefunctions of fermion zero modes, both of which give rise to more than an order-of-magnitude effect on the couplings. There are also effects coming from fermion boundary mixing terms (Eq. (26) ), which will introduce only order one uncertainty in our estimates. However, the dependence of the couplings on the parameters $\theta_{H}$ and wavefunctions of fermion zero modes should be robust against the effects from these mixing terms.

A comment is in order here about the region of parameter space we are considering. As pointed out in [16, 8], a light $t^{(1)}$ (first KK mode of top quark) is a promising signature for GHU. We will see later that a light $t^{(1)}$ is also desirable for the collider study of the coset gauge bosons. We generically get two light $t^{(1)}$ states in the regions of parameter space when $c_{1}<0$. In this case, the $\mathrm{SM}(t, b)_{L}$ profile is highly peaked near the $\mathrm{TeV}$ brane and thus the $\mathrm{SM} t_{R}$ is less so (in order to obtain the correct top quark mass). We find that one of the light $t^{(1)}$ states is mostly $S U(2)_{L}$ singlet in this case. Thus, the coupling of SM bottom (doublet) and this light $t^{(1)}$ to the coset gauge boson (doublet) is large since it is allowed by the quantum numbers (i.e., no need for EWSB) and is not suppressed by profiles either. This coupling can then give a significant contribution to the production of the coset gauge boson. Therefore, we focus on this region of parameter space. We will often denote this singlet light $t^{(1)}$ as "the light $t^{(1)}$ " in what follows. To simplify notation, we will also use $t, b$ to denote SM top and bottom fermions when there is no confusion.

References [6] showed that the one loop contributions of such light $t^{(1)}$ states to the $T$ parameter and to the shift in $Z b \bar{b}$ coupling can be consistent with the data. Another potential constraint comes from the shift in the $W t b$ coupling. We have numerically studied the shift in the $t b W$ coupling induced by mixing of zero and KK modes of both $W$ and top (including the effect of the light $t^{(1)}$ state). We find that this shift is smaller than $\sim 10 \%$, as required by the recent measurements at Tevatron [28].

Alternatively, the SM $t_{R}$ can be highly peaked near the $\mathrm{TeV}$ brane [and the $\mathrm{SM}(t, b)_{L}$ less so], which results in the light $t^{(1)}$ being a doublet [16] and a large coset- $t_{R^{-}} t^{(1)}$ coupling. However, the top quark content of the proton is negligible (cf. bottom quark content which is larger) so that this coupling will not be that useful for production of coset gauge bosons.

\section{- Charged Coset Gauge Bosons $\left(W_{C}\right)$}

\footnotetext{
${ }^{14}$ The coupling between coset gauge bosons and two SM gauge bosons are not studied here since they vanish at leading order in Higgs vev due to quantum number (as argued in Section 3) and thus are not relevant for collider study.
} 
- $g_{W_{C} t b}$ : coupling between coset $W_{C}$, SM top and SM bottom. We first discuss the coupling for left-handed fermions. Once the Higgs boson gets a vev, there will be mixing between $W_{L}$ and $W_{C}$ and between $t_{1 L}$ and $\hat{t}_{1 L}$. From another point of view, this mixing comes from the gauge transformation (Eqs. (28) and (30)) that link the wavefunctions with vanishing Higgs vev and wavefunctions with non-vanishing Higgs vev. For example, from Eq. (86) we can see the wavefunction of $W_{L}$ with non-vanishing Higgs vev contains some part of $W_{C}$ wavefunction with vanishing Higgs vev, and the amount is $\frac{\sin \theta_{H}}{\sqrt{2}}$. Therefore the dominant contribution to the coupling comes from the following overlap integral of wavefunctions

$$
g_{W_{C} t_{L} b_{L}} \approx-\frac{g_{5}}{\sqrt{2}} \int \frac{d z}{z^{4}}\left[f_{W_{L}} F_{t_{1}} F_{b_{1}}+f_{W_{C}} F_{\hat{t}_{1}} F_{b_{1}}\right]
$$

The first term comes from the mixing between $W_{L}$ and $W_{C}$, and the second term comes from the mixing between $t_{1 L}$ and $\hat{t}_{1 L}$. Here we have assumed that the zero mode $t_{L}, b_{L}$ lives mainly in the first fermion multiplet $\Psi_{1}$. This happens when $c_{1}<0$. To estimate this coupling, we need to know the wavefunctions of $t_{L}, b_{L}$ and $W_{C}$. The wavefunctions of $W_{C}$ are peaked near the IR brane (i.e., their size at the IR brane is $\sim O(1)$ in units of $\sqrt{k}$ ) since they are KK modes, and each of the wavefunctions of $t_{L}$ and $b_{L}$ at the IR brane is $f\left(c_{1}\right) \approx \sqrt{\frac{1}{2}-c_{1}}$ (in units of $\sqrt{k}$ ). Finally, the overlap integral will be dominated by a region of size $\sim \frac{1}{k}$ near the IR brane. This gives us an estimate

$$
\left|g_{W_{C} t_{L} b_{L}}\right| \sim \frac{g_{5} \sqrt{k}}{\sqrt{2}}\left(\frac{1}{2}-c_{1}\right) \frac{\sin \theta_{H}}{\sqrt{2}}
$$

where $\frac{\sin \theta_{H}}{\sqrt{2}}$ comes from mixing induced by Higgs vev. From Eq. (33) we can see that it is possible to get order one coupling between $W_{C}$ and SM top and bottom quarks 15 The right-handed coupling $g_{W_{C} t_{R} b_{R}}$ should be much smaller than the left-handed coupling $g_{W_{C} t_{L} b_{L}}$ since the wavefunction of $b_{R}$ is much smaller than that of $b_{L}$ near the IR brane. Therefore, it is irrelevant for collider study.

- $g_{W_{C} t^{(1)} b}$ : coupling between coset $W_{C}$, first top KK mode, SM bottom quark. We first study the left-handed coupling. Note that the $t^{(1)}$ is mostly $S U(2)_{L}$ singlet and its wavefunction is also peaked near the IR brane (i.e., its size at the IR brane is $\sim O(1)$ in units of $\sqrt{k}$ ), just like $W_{C}$. Thus the size of this coupling should be controlled simply by the single $b$ wavefunction near the IR brane (i.e., no factor of EWSB). Therefore the coupling should be of order $\frac{g_{5} \sqrt{k}}{\sqrt{2}} f\left(c_{1}\right) \approx \frac{g_{5} \sqrt{k}}{\sqrt{2}} \sqrt{\frac{1}{2}-c_{1}}$ in the $\theta_{H} \rightarrow 0$ limit. Including the effect of nonzero Higgs

\footnotetext{
${ }^{15}$ Clearly, the coupling analogous to Eq. (33) is negligible for light left/right-handed SM fermions which have $c>(<) 1 / 2(-1 / 2)$ and hence $f(c) \ll 1$. In particular, the coset gauge boson wavefunctions vanish near the Planck brane so that the wavefunction overlap comes only from near the TeV brane, unlike for $\mathrm{KK} W / Z$ where the flavoruniversal part of the coupling to two SM fermions comes from overlap near the Planck brane.
} 
vev will only give a small correction to this coupling, thus the estimate remains the same. The coupling for right-handed fermions should be much smaller due to the same reason that the wavefunction of $b_{R}$ near the IR brane is small.

- $g_{W_{C} t^{(1)} b^{(1)}}$ : coupling between coset $W_{C}$, first top KK mode and first bottom KK mode. Since the KK modes of fermions are localized near the IR brane, the coupling for both left-handed and right-handed fermions should be of order $\frac{g_{5} \sqrt{k}}{\sqrt{2}}$ (i.e., no suppression due to profiles or EWSB), up to order one coefficients coming from boundary mixing terms.

- $\underline{\text { Neutral Coset Gauge Boson }\left(Z_{C}\right)}$

- $g_{Z_{C} t t}$ : coupling between neutral coset gauge boson $Z_{C}$ and SM top quark. For left-handed coupling, the estimate is similar to that of $g_{W_{C} t_{L} b_{L}}$ :

$$
g_{Z_{C} t_{L} t_{L}} \sim g_{5} \sqrt{k}\left(\frac{1}{2}-c_{1}\right) \frac{\sin \left(\theta_{H}\right)}{\sqrt{2}}
$$

For right-handed coupling, the estimate is also similar

$$
g_{Z_{C} t_{R} t_{R}} \sim g_{5} \sqrt{k}\left(\frac{1}{2}+c_{2}\right) \frac{\sin \left(\theta_{H}\right)}{\sqrt{2}}
$$

- $g_{Z_{C} t^{(1)} t}$ : coupling between coset $Z_{C}$, KK top and SM top quark. For left-handed coupling the estimate is

$$
g_{Z_{C} t_{L}^{(1)} t_{L}} \sim g_{5} \sqrt{k} \sqrt{\frac{1}{2}-c_{1}}
$$

and for right-handed coupling

$$
g_{Z_{C} t_{R}^{(1)} t_{R}} \sim g_{5} \sqrt{k} \sqrt{\frac{1}{2}+c_{2}}
$$

- $g_{Z_{C} t^{(1)} t^{(1)}}$ : coupling between coset $Z_{C}$ and KK top quark. Since $Z_{C}$ always couples to two fermions transforming in different representation of $S U(2)_{L}$, this coupling will not be generated in the $\theta_{H} \rightarrow 0$ limit. Therefore, a rough estimate of this coupling is

$$
g_{Z_{C} t^{(1)} t^{(1)}} \sim g_{5} \sqrt{k} \frac{\sin \theta_{H}}{\sqrt{2}}
$$

This estimate holds for both left-handed and right-handed couplings since the wavefunctions for $t_{L}^{(1)}$ and $t_{R}^{(1)}$ are both IR localized.

- $g_{Z_{C} b b}$ : coupling between neutral coset gauge boson $Z_{C}$ and SM bottom quark. For the lefthanded coupling, naive estimate will give us

$$
g_{Z_{C} b_{L} b_{L}}^{\text {naive }} \sim g_{5} \sqrt{k}\left(\frac{1}{2}-c_{1}\right) \frac{\sin \theta_{H}}{\sqrt{2}}
$$


However, this coupling is very small (i.e., not relevant for collider signals) due to custodial symmetry. To be specific, the two contributions to this coupling coming from $Z_{C}$ mixing with $W_{L, R}^{3}$ cancel each other. Similarly, the contributions to this coupling coming from $(2,2)$ fermion mixing with $(1,3)$ and $(3,1)$ fermion cancel each other. This cancellation is related to the build-in custodial symmetry of the model that protects the $g_{Z b_{L} b_{L}}$ coupling (see Appendix C for more detailed discussion). Note that this cancelation does not happen for top quark since its $W_{L}^{3}$ and $W_{R}^{3}$ charges are different. The right-handed coupling is small due to the small $b_{R}$ wavefunction near IR brane.

- $g_{Z_{C} b b^{(1)}}$ : coupling between coset $Z_{C}$, SM bottom and KK bottom quark. The left-handed coupling is small due to similar cancelation that suppress $g_{Z_{C} b_{L} b_{L}}$ coupling. The right-handed coupling is also small because of the small $b_{R}$ wavefunction near IR brane.

There is an additional coset gauge boson $A_{\mu}^{\hat{4}}$ (gauge boson of the generator $T^{\hat{4}}$ ) which is the vector partner of physical Higgs boson. We do not consider it here because its coupling with two SM fermions vanishes 16 Even though it has nonzero coupling with $b b^{(1)}$ and $t t^{(1)}$, its production at the LHC is still suppressed. The reason is that the $b^{(1)}$ is not light (in the case of associated production with $b^{(1)}$ using the coupling to $\left.b b^{(1)}\right)$ and the top quark content of the proton is negligible, even though $t^{(1)}$ is light (in the case of associated production with $t^{(1)}$ using the coupling to $t t^{(1)}$ ).

We can compare the pattern of couplings estimated here with our conclusion using the two-site approach. In fact, there is a one-to-one correspondence/dictionary between two-site language and warped extra dimension models (see [7, 32]):

$$
\begin{aligned}
\text { SM states } & \leftrightarrow \text { zero modes } \\
\text { heavy states } & \leftrightarrow \text { KK modes } \\
\sin \theta_{\psi_{L, R}} & \leftrightarrow f\left(c_{L, R}\right), \\
\sin \theta_{G} & \leftrightarrow \frac{1}{\sqrt{k L}} \\
g_{*} & \leftrightarrow g_{5} \sqrt{k}
\end{aligned}
$$

Based on this identification, we can see that the estimates for specific $5 \mathrm{D}$ model agree with those obtained using two-site description, the latter estimates being applicable to the general warped/composite PGB Higgs framework. Namely, the coset gauge boson generally couple strongly with SM fermions (zero modes) and heavy fermions (KK modes). We emphasize again that the conclusions above for the 5D model are rough, but are quite general, for example, they do not

\footnotetext{
${ }^{16}$ The reason for this is that Higgs vev does not induce an effect on $A_{\mu}^{\hat{4}}$ coupling since the gauge transformation (Eq. (29) ) commutes with $T^{\hat{4}}$.
} 
depend on whether the bulk gauge symmetry breaking $[S O(5) \rightarrow S O(4)]$ vev is infinite (as in GHU models) or finite17. We will further validate these estimates by computing them numerically for the specific 5D GHU model in section 4.5.

\subsubsection{Exact Couplings}

The exact couplings of coset gauge bosons can be obtained by overlap integrals of wavefunctions. We define gauge boson wavefunction matrix

$$
G(z, v) \equiv f^{a_{L}}(z, v) T^{a_{L}}+f^{a_{R}}(z, v) T^{a_{R}}+f^{\hat{a}}(z, v) T^{\hat{a}}+f^{\hat{4}}(z, v) T^{\hat{4}} .
$$

And we just use $F_{1,2,3}^{\Psi}(z, v)$ (see Eq. (30) ) to denote the wavefunctions of the three fermionic multiplets. Then we can get the coupling between fermions and coset gauge bosons:

$g_{G F F}=\int \frac{d z}{(k z)^{4}}\left\{g_{5}\left[F_{1,2}^{\Psi \dagger} A G A^{\dagger} F_{1,2}^{\Psi}+\operatorname{Tr}\left(F_{3}^{\Psi \dagger}\left[G, F_{3}^{\Psi}\right]\right)\right]+g_{X}\left[f^{X}\left(F_{1,2}^{\Psi \dagger} F_{1,2}^{\Psi}+\operatorname{Tr}\left[F_{3}^{\Psi \dagger} F_{3}^{\Psi}\right]\right)\right]\right\} \cdot(4$

where matrix $A$ is defined in Eq. (22). We use this formula to do numerical analysis in the next section.

\subsection{Numerical Results}

The purpose of our numerical scan is to find some points in the parameter space of the GHU model, in particular, specific values of the coset gauge boson couplings, which can then be used as benchmarks for our collider study. Therefore, we pick a specific model of GHU introduced in [8] to do our numerical scan. However, we should not treat the results as being model dependent for the following reason. As we argued in previous sections, the masses of coset gauge bosons in non-minimal models can be different from minimal GHU models. However, the couplings of coset gauge bosons are not sensitive to this non-minimal structure since their pattern is determined by the quantum numbers and the fact that coset gauge boson wavefunctions are peaked near the IR brane.

In our numerical scan, we fix $k=10^{18} \mathrm{GeV}$ and we scanned over the input parameters $g_{5} \sqrt{k}, k L, c_{1,2,3}, M_{B 1, B 2}$. Even though for minimal model we have $g_{5} \sqrt{k} \approx g \sqrt{k L}$, this relationship between $5 \mathrm{D}$ coupling and $4 \mathrm{D}$ gauge coupling is modified once we include brane kinetic terms for gauge fields. Therefore, we choose to scan $g_{5} \sqrt{k}$ over the range $[g \sqrt{k L}, 2 g \sqrt{k L}]$. We calculate the Higgs potential (Eq. (31)) and minimize it to find the Higgs vev $v$. We can then calculate the particle spectrum using the spectral functions (Eqs. (105), (115), (131) and (132)). We collect points with reasonable top and $W / Z$ masses. Finally, the couplings of coset gauge bosons are calculated using Eq. (42). The important couplings are:

\footnotetext{
${ }^{17}$ This insensitivity is related to a similar one in the two-site description in section 3 , in the latter case to $f_{\phi}$, the scalar vev breaking the global symmetry $[S O(5) \rightarrow S O(4)]$ in the composite sector.
} 
(i) for charged coset gauge boson $W_{C}$

$$
\begin{aligned}
\mathcal{L}_{W_{C}} & =g_{W_{C} t_{L} b_{L}} \bar{t}_{L} \gamma_{\mu} b_{L} W_{C}^{+\mu}+g_{W_{C} t_{R} b_{R}} \bar{t}_{R} \gamma_{\mu} b_{R} W_{C}^{+\mu} \\
& +g_{W_{C} t_{L}^{(1)} b_{L}} \bar{t}_{L}^{(1)} \gamma_{\mu} b_{L} W_{C}^{+\mu}+g_{W_{C} t_{R}^{(1)} b_{R}} \bar{t}_{R}^{(1)} \gamma_{\mu} b_{R} W_{C}^{+\mu}+\text { h.c. },
\end{aligned}
$$

(ii) for neutral coset gauge boson $Z_{C}$

$$
\begin{aligned}
\mathcal{L}_{Z_{C}} & =g_{Z_{C} t_{L} t_{L}} \bar{t}_{L} \gamma_{\mu} t_{L} Z_{C}^{\mu}+g_{Z_{C} t_{R} t_{R}} \bar{t}_{R} \gamma_{\mu} t_{R} Z_{C}^{\mu} \\
& +g_{Z_{C} t_{L}^{(1)} t_{L}} \bar{t}_{L}^{(1)} \gamma_{\mu} t_{L} Z_{C}^{\mu}+g_{Z_{C} t_{R}^{(1)} t_{R}} \bar{t}_{R}^{(1)} \gamma_{\mu} t_{R} Z_{C}^{\mu} \\
& +g_{Z_{C} b_{L} b_{L}} \bar{b}_{L} \gamma_{\mu} b_{L} Z_{C}^{\mu}+g_{Z_{C} b_{R} b_{R}} \bar{b}_{R} \gamma_{\mu} b_{R} Z_{C}^{\mu} \\
& +g_{Z_{C} b_{L}^{(1)} b_{L}} \bar{b}_{L}^{(1)} \gamma_{\mu} b_{L} Z_{C}^{\mu}+g_{Z_{C} b_{R}^{(1)} b_{R}} \bar{b}_{R}^{(1)} \gamma_{\mu} b_{R} Z_{C}^{\mu}+\text { h.c. },
\end{aligned}
$$

(iii) for first KK top $t^{(1)}$

$$
\begin{aligned}
\mathcal{L}_{t^{(1)}} & =g_{W t_{L}^{(1)} b_{L}} \bar{t}_{L}^{(1)} \gamma_{\mu} b_{L} W^{+\mu}+g_{W t_{R}^{(1)} b_{R}} \bar{t}_{R}^{(1)} \gamma_{\mu} b_{R} W^{+\mu} \\
& +g_{Z t_{L}^{(1)} t_{L}} \bar{t}_{L}^{(1)} \gamma_{\mu} t_{L} Z^{\mu}+g_{Z t_{R}^{(1)} t_{R}} \bar{t}_{R}^{(1)} \gamma_{\mu} t_{R} Z^{\mu} \\
& +g_{H t_{L}^{(1)} t_{R}} \bar{t}_{L}^{(1)} h t_{R}+g_{H t_{R}^{(1)} t_{L}} \bar{t}_{R}^{(1)} h t_{L}+\text { h.c. },
\end{aligned}
$$

\begin{tabular}{|c|c|c|c|c|c|c|c|}
\hline & $g_{W_{C} t_{L} b_{L}}$ & $g_{W_{C} t_{R} b_{R}}$ & $g_{W_{C} t_{L}^{(1)} b_{L}}$ & \multirow{2}{*}{$\begin{array}{c}g_{W_{C} t_{R}^{(1)} b_{R}} \\
0.00207\end{array}$} & & \\
\hline & & 0.712 & 0.00169 & -1.945 & & & \\
\hline$g_{Z_{C} t_{L} t_{L}}$ & $g_{Z_{C} t_{R} t_{R}}$ & $g_{Z_{C} t_{L}^{(1)} t_{L}}$ & $g_{Z_{C} t_{R}^{(1)} t_{R}}$ & $g_{Z_{C} b_{L} b_{L}}$ & $g_{Z_{C} b_{R} b_{R}}$ & $g_{Z_{C} b_{L}^{(1)} b_{L}}$ & $g_{Z_{C} b_{R}^{(1)} b_{R}}$ \\
\hline-0.930 & 0.119 & 1.242 & 0.177 & 0.0235 & -0.0219 & 0.0294 & 0.136 \\
\hline & $g_{W t_{L}^{(1)} b_{I}}$ & $g_{W t_{R}^{(1)} b_{I}}$ & $g_{Z t_{L}^{(1)} t_{L}}$ & $g_{Z t_{R}^{(1)} t_{R}}$ & $g_{H t_{L}^{(1)} t_{R}}$ & $g_{H t_{R}^{(1)} t_{L}}$ & \\
\hline
\end{tabular}

where the subscripts $L, R$ imply the chirality of the fermion. We present here a sample point with the couplings from the scan in Table 1 and Table 2. This will be served as benchmark point for collider study.

\begin{tabular}{|c|c|c|c|c|c|c|c|}
\hline$k e^{-k L}$ & $g_{5} \sqrt{k}$ & $c_{1}$ & $c_{2}$ & $c_{3}$ & $M_{B 1}$ & $M_{B 2}$ & $\theta_{H}$ \\
\hline $956 \mathrm{GeV}$ & 7.16 & -0.364 & -0.446 & -0.559 & 1.419 & -0.139 & 0.410 \\
\hline
\end{tabular}

Table 1: Input parameters for sample points used in our numerical calculation. We fix $k=$ $10^{18} \mathrm{GeV} . c_{1,2,3}$ are the bulk mass parameters for the fermionic multiplets. $M_{B 1}, M_{B 2}$ are boundary mass parameters needed to get correct SM fermion masses (see Eq. 26).

Table 2: Numerical values of the couplings for the sample point choice. 

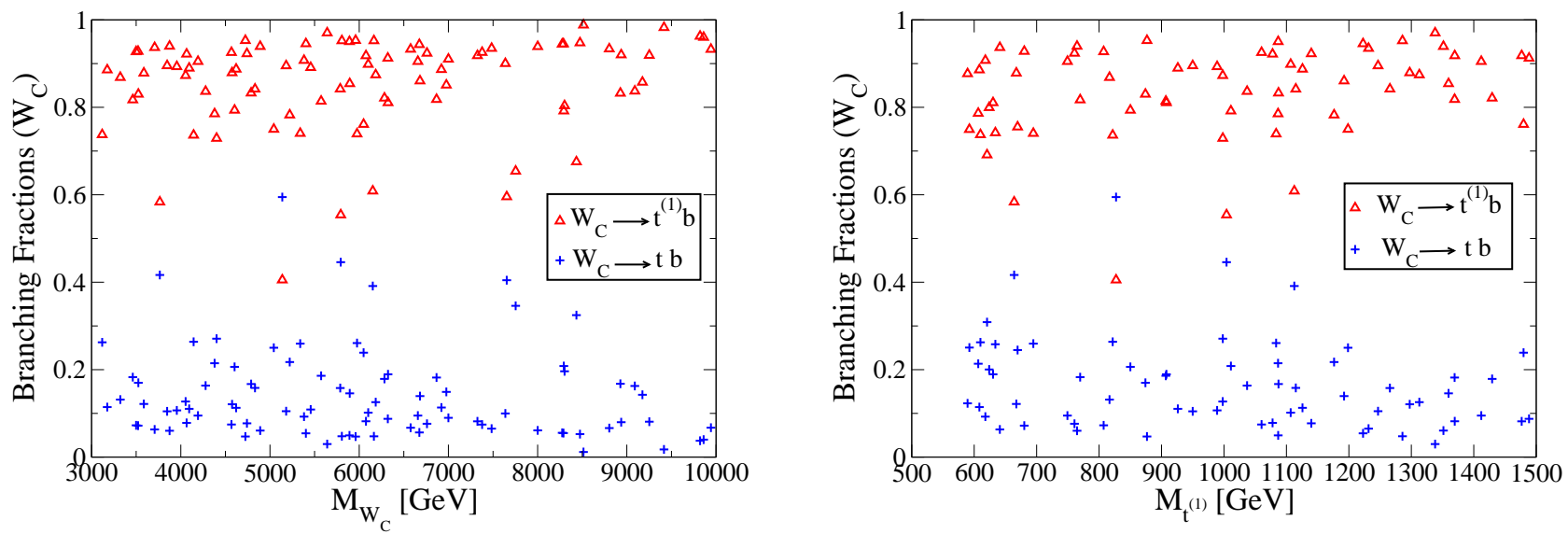

Figure 3: Scatter plots for the branching fractions $\operatorname{Br}\left(W_{C} \rightarrow t^{(1)} b\right)$ (triangle symbol) and $\operatorname{Br}\left(W_{C} \rightarrow\right.$ $t b)$ (cross symbol) versus (a) $M_{W_{C}}$ and (b) $M_{t^{(1)}}$, respectively.

\section{$5 \quad$ LHC signals}

As discussed in sections 3 and 4 , the most characteristic feature of coset gauge bosons is that they are vector bosons possessing Higgs quantum number. This uniquely fixes the pattern they are coupled to SM particles and other KK modes - they predominantly couple to one SM and one KK fermions as their couplings to a pair of SM particles are only induced by EWSB and are thus subdominant. This further determines how they are produced and decay at the LHC.

As will be discussed in section [5.2, the production rate of neutral coset KK modes at LHC is very low in general. We will thus focus on the LHC signals of charged coset KK modes $W_{C}$. Our study is based on a set of points in the parameter space that give reasonable SM particle masses and generate EWSB radiatively as discussed in section 4.5. We first discuss the decay of charged coset gauge KK boson in 5.1 and its production at the LHC in 5.2 using this set of points. We then use couplings corresponding to the representative benchmark point in Table 2 and discuss in detail the signal and background at the LHC in 5.3 .

\section{$5.1 \quad$ Decay of $W_{C}$}

There are following decay channels of $W_{C} 18$

$$
W_{C} \rightarrow t b^{(1)}, t^{(1)} b, t b
$$

Compared to $W_{C} \rightarrow t^{(1)} b$, the branching fraction of $W_{C} \rightarrow t b^{(1)}$ is substantially suppressed due to kinematical reasons. It is one of the important properties of GHU models that there exists a light

\footnotetext{
${ }^{18}$ The other decay channels involving light SM fermions are negligible.
} 


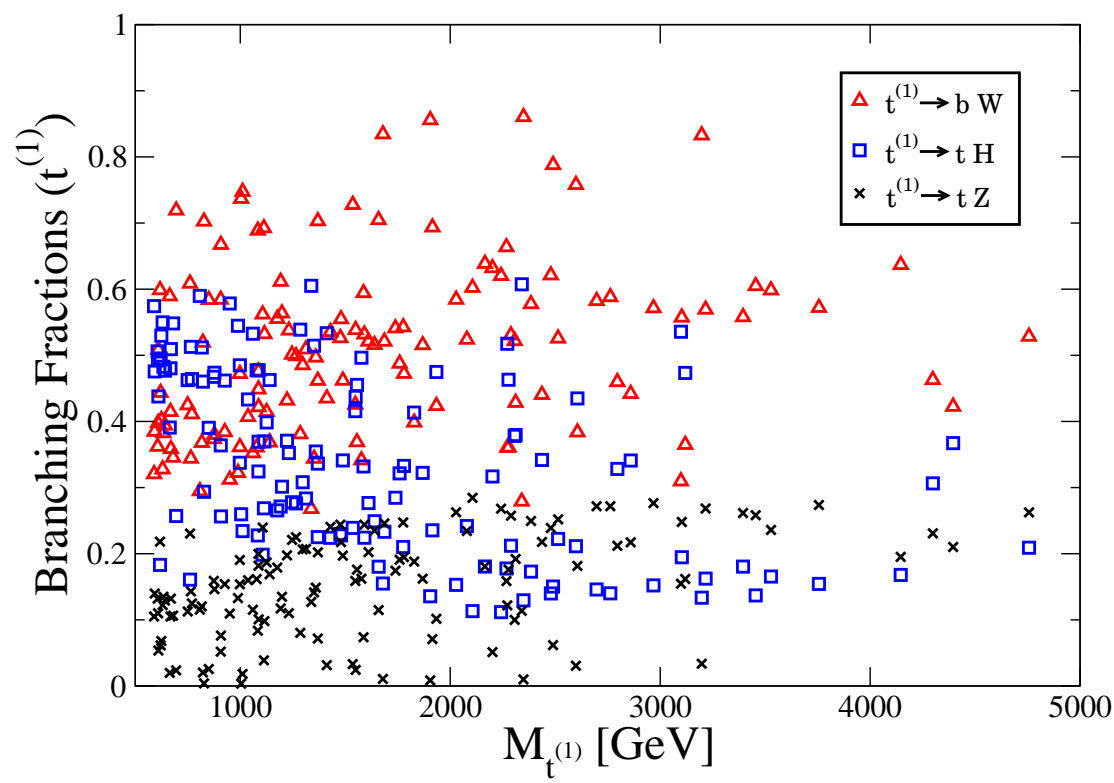

Figure 4: Scatter plots for the branching fractions $\operatorname{Br}\left(t^{(1)} \rightarrow W b\right)$ (triangle symbol), $\operatorname{Br}\left(t^{(1)} \rightarrow t h\right)$ (square symbol), and $\operatorname{Br}\left(t^{(1)} \rightarrow t Z\right)$ (cross symbol) versus $M_{t^{(1)}}$.

KK mode of top quark $t^{(1)}$ [8]. The mass of $b$-quark KK mode $b^{(1)}$ is, on the other hand, usually much heavier. Thus the decay $W_{C} \rightarrow t b^{(1)}$ is in most cases highly suppressed, if not forbidden. In the following, for simplicity and without losing the general feature, we will assume $b^{(1)}$ is heavier than $W_{C}$, forbidding this decay channel completely.

On the other hand, the branching fraction of $W_{C} \rightarrow t b$ is much suppressed compared to $W_{C} \rightarrow$ $t^{(1)} b$ due to dynamical reasons. As discussed in sections 3 and 4 , the quantum number of $W_{C}$ forbids its coupling to SM quarks like $\bar{t} b$ at leading order. This coupling is only induced by Higgs vev after EWSB and is thus suppressed by $v / f_{h}$. This determines the typical trend of branching fractions of $W_{C} \rightarrow t^{(1)} b$ and $W_{C} \rightarrow t b$ decays, shown in Fig. 3 ,

Since $t^{(1)}$ appears in the most dominant decay channel of $W_{C}$, we thus comment on $t^{(1)}$ decay next. There are three decay channels of $t^{(1)}$

$$
t^{(1)} \rightarrow b W, \quad t h, \quad t Z \text {. }
$$

This has been studied in great detail in Ref. [17, where it has been pointed out that, for large $M_{t^{(1)}}$, the branching fractions should follow the relation $2: 1: 1$, according to the Goldstone Boson Equivalence Theorem, where as for small $M_{t^{(1)}}$, this ratio does not hold. In Ref. [17], the branching fractions are only shown for $M_{t^{(1)}}$ larger than $1 \mathrm{TeV}$. Since our primary goal is to explore the reach 


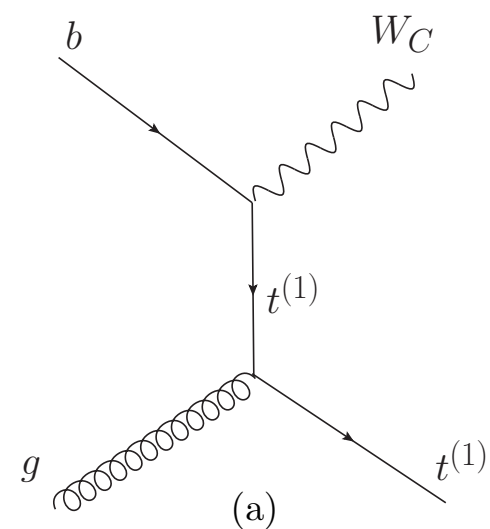

(a)

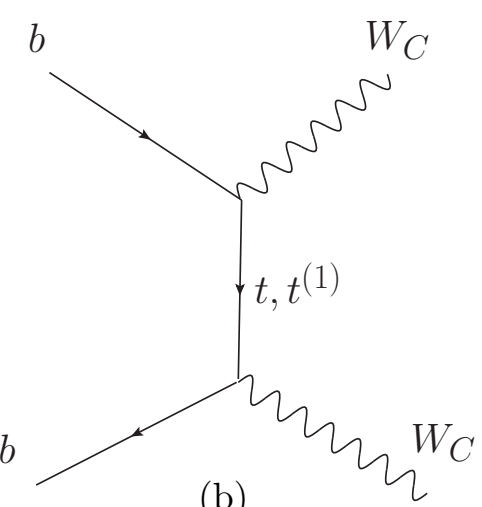

(b)

Figure 5: Representative Feynman diagrams for (a) $W_{C} t^{(1)}$ associated production and (b) $W_{C} W_{C}$ pair production.

of LHC on discovering $W_{C}$, a relatively light $t^{(1)}$ would be more relevant. We thus extend the $t^{(1)}$ decay branching fractions to a wider range of $500 \mathrm{GeV}-5 \mathrm{TeV}$, as in Fig. 4. The 2:1:1 ratios hold for $M_{t^{(1)}}>3 \mathrm{TeV}$. For a light $t^{(1)}, M_{t^{(1)}}<1 \mathrm{TeV}$, the branching fractions of $t^{(1)} \rightarrow b W$ and $t^{(1)} \rightarrow t h$ are close and both are significantly larger than that of $t^{(1)} \rightarrow t Z$. The LHC search of $t^{(1)}$ has also been discussed in Ref. [17] and positive conclusions were reached. We therefore assume that $t^{(1)}$ has been observed with its mass approximately known a priori to the searches for $W_{C}$.

\subsection{Coset KK modes production at the LHC}

The coset gauge bosons, as all the KK modes in general, have profiles with large overlap with the third generation SM fermions $(t, b)$ and hence couple more strongly to them as compared to the 1st/2nd generation SM quarks. However, the dominant production of $\mathrm{KK} W / Z$ is still (typically) via $u$ and $d$ quarks. On the other hand, the coupling to light quarks is smaller for the case of coset gauge bosons than the $\mathrm{KK} W / Z$ (as discussed in section 3). This feature motivates consideration of coupling of coset gauge bosons to bottom quarks for their production at the LHC. From the discussion in section 4.4, and as shown explicitly in Table 2, the neutral coset KK modes $Z_{C}$ couple strongly to $t(\bar{t})$, but rather weakly to $b(\bar{b})$, indicating that its production is highly suppressed at the LHC. We will then focus on the production of charged ones $W_{C}^{ \pm}$in the following. Figures 5 and 6 show the representative Feynman diagrams for the $W_{C}$ associated production with a new heavy particle and with a SM particle, respectively. Between the two mechanisms (associated and pair) for production in Fig. 5, the production rate $b g(\bar{b} g) \rightarrow W_{C}^{ \pm} t^{(1)}$ is clearly higher than that of $b \bar{b} \rightarrow W_{C}^{+} W_{C}^{-}$, due to its lower kinematical threshold and higher gluon luminosity in the proton. A similar argument also applies in Fig. [6 in favor of the production $b g \rightarrow W_{C}^{ \pm} t$.

In Fig. 7, we show the total cross sections for these two processes $b g \rightarrow W_{C}^{ \pm} t^{(1)}, W_{C}^{ \pm} t$, as a function of their masses (a) $M_{W_{C}}$ and (b) $M_{t^{(1)}}$. The t-channel contribution dominates over the 


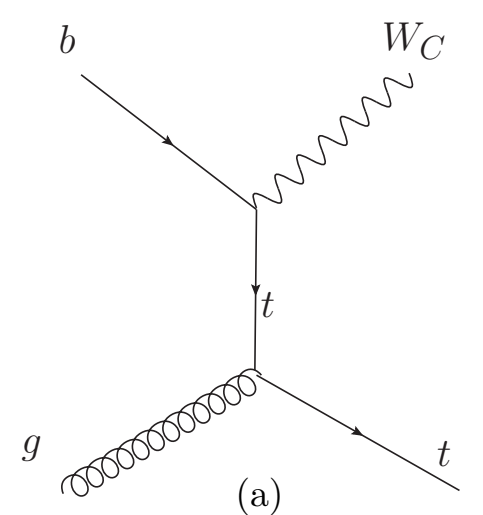

(a)

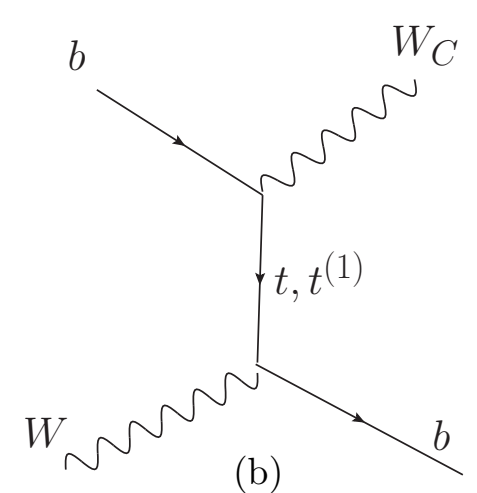

(b)

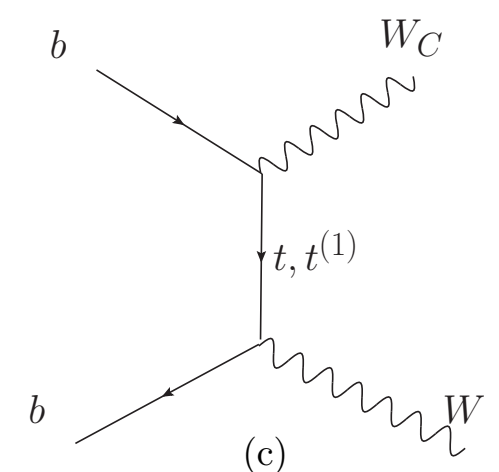

(c)

Figure 6: Representative Feynman diagrams for associated production (a) $W_{C} t$, (b) $W_{C} b$, and (c) $W_{C} W$, respectively.

s-channel one. We turn off small couplings $g_{W_{C} t_{R}^{(1)} b_{R}}$ and $g_{W_{C} t_{R} b_{R}}$, fix the relative size of couplings as $g_{W_{C} t_{L}^{(1)} b_{L}} / g_{W_{C} t_{L} b_{L}}=5$, and factor out the order one coupling $g_{W_{C} t_{L}^{(1)} b_{L}}$. Comparing these two processes, we see that $W_{C}^{ \pm} t^{(1)}$ production wins due to the stronger coupling as in Fig. 7(a); while $W_{C}^{ \pm} t$ production wins for the phase space when $M_{t(1)}>1 \mathrm{TeV}$, as in Fig. 7 (b). The cross sections can be typically of the order of a fraction of $\mathrm{fb}$ in the mass range of our interest. Since our goal is to explore the reach of LHC on discovering $W_{C}$, we will focus on the low mass region of $M_{t^{(1)}}$, where the associated production $b g(\bar{b} g) \rightarrow W_{C}^{ \pm} t^{(1)}$ dominates among the various non-resonant production channels.

We estimate, based on appropriate rescaling of numbers in Fig. 4 (a) of 1st reference in [30] or Fig. 7 of reference [29] for example, that the resonant production of coset gauge bosons via light quarks might be comparable to the above associated production, but the former channel suffers (as discussed at the end of section (3) from a pollution from production of the KK $W$. On the other hand, it is easy to see that a similar pollution for associated $W_{C}$ production is negligible: note that (as discussed earlier) KK $W$ does not couple to $b_{L}$ (doublet) and light $t^{(1)}$ (singlet) before EWSB, i.e., the pollution from KK $W$ in this channel is suppressed by EWSB 19 So, we will consider only associated production of $W_{C}$ in this paper. For the purpose of illustration, we choose

$$
M_{W_{C}}=2 \mathrm{TeV}, \quad M_{t^{(1)}}=500 \mathrm{GeV}
$$

\footnotetext{
${ }^{19}$ The coupling of KK $W_{R}$, i.e., the charged gauge boson of $S U(2)_{R}$, to $t_{L}^{(1)}$ and $b_{L}$ is similarly suppressed compared to that of $W_{C}$ to $t_{L}^{(1)}$ and $b_{L}$, again since $t^{(1)}$ is mostly singlet of $S U(2)_{L} \times S U(2)_{R}$ in the part of parameter space we are considering. Actually, there is another top KK mode living in the bi-doublet representation of $S U(2)_{L} \times S U(2)_{R}$, which has a coupling to $W_{R}$ (but not to KK $W_{L}$ ) and $b_{L}$ which is similar in size to the coupling of coset gauge bosons to $b_{L}$ and the singlet $t^{(1)}$. However, the mass of this bi-doublet KK top is $\sim 1.4$ times higher than that of the singlet $t^{(1)}$ for the point we are considering, leading to the cross section for associated production of $W_{R}$ (via exchange of the bi-doublet top KK mode) being suppressed (relative to that for coset gauge boson with singlet top KK mode exchange), for the case when $W_{R}$ and $W_{C}$ have the same masses. Again, compare this situation to the pollution encountered in the resonant production of coset gauge bosons mentioned above.
} 

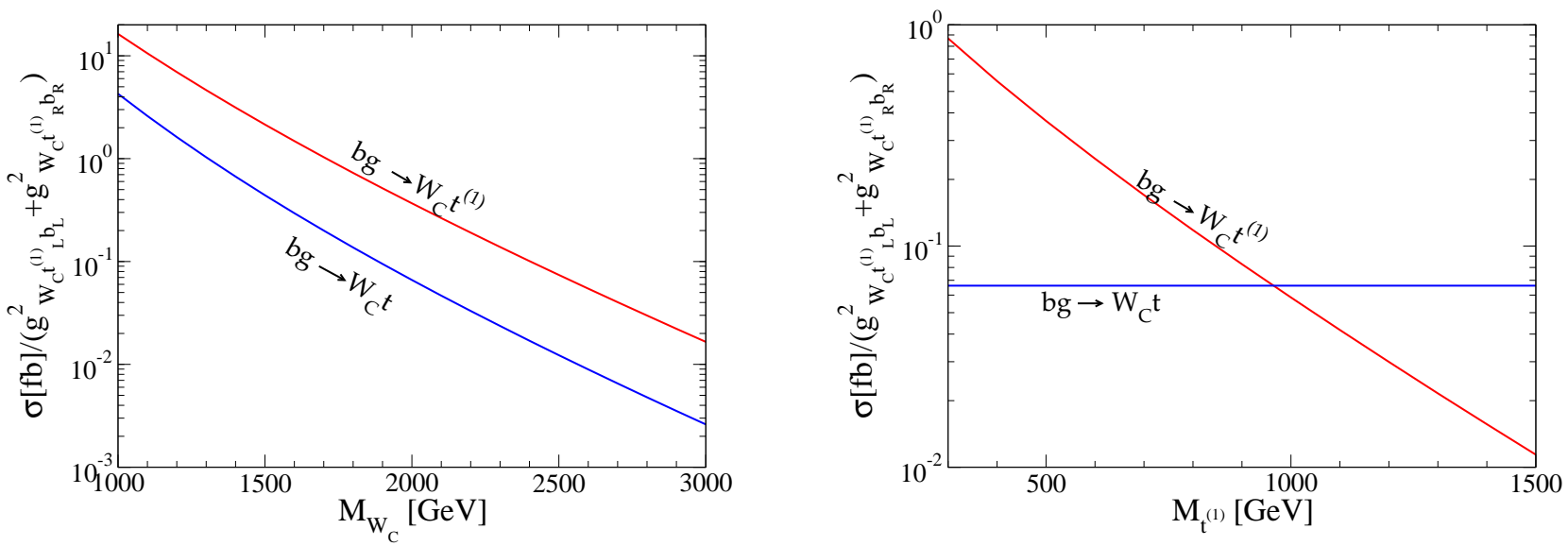

Figure 7: Cross section at the LHC $(14 \mathrm{TeV})$ for $p p \rightarrow W_{C}^{ \pm} t^{(1)}, W_{C}^{ \pm} t$ versus their masses (a) $M_{W_{C}}$ and (b) $M_{t^{(1)}}$. The coupling ratio $g_{W_{C} t_{L}^{(1)} b_{L}} / g_{W_{C} t_{L} b_{L}}=5$ is fixed. The small couplings $g_{W_{C} t_{R}^{(1)} b_{R}}$ and $g_{W_{C} t_{R} b_{R}}$ are turned off, and order-one coupling square $g_{W_{C} t_{L}^{(1)} b_{L}}^{2}$ is factored out.

as the reference point, and explore the dependence on the masses later.

\subsection{Search of $W_{C}$ at LHC: Signals and Backgrounds}

To further quantify the search of $W_{C}$ at the LHC, we fix to one point in the parameter space and study the signals and background in detail. The couplings corresponding to this parameter point is shown in Table 2, We use the $M_{W_{C}}$ and $M_{t^{(1)}}$ as in Eq. (48). The cases with other couplings and masses can be estimated by a proper scaling according to the production cross section shown in Fig. 7. We use the CTEQ6.1L parton distribution functions [33]. We concentrate on the dominant channels of production of $W_{C}$ and its decay

$$
b g \rightarrow W_{C} t^{(1)} \rightarrow b t^{(1)} t^{(1)} .
$$

We consider all the decay channels of $t^{(1)}$ as in Eq. (47), which result in different signals, as we will study in detail below.

\subsection{1 $\quad t^{(1)} t^{(1)} \rightarrow b W, b W$}

We first consider the case with both $t^{(1)}$ 's decaying to $b W$ :

$$
b g \rightarrow W_{C} t^{(1)} \rightarrow b+2 t^{(1)} \rightarrow 3 b+2 W
$$


whose branching fraction is a product of three factor: 20

$$
\operatorname{Br}\left(W_{C} \rightarrow b t^{(1)}\right) \times B r\left(t^{(1)} \rightarrow b W\right)^{2} \approx 90 \% \times(50 \%)^{2}=22.5 \%
$$

We consider the semileptonic decays of $2 W^{\prime}$ 's where one $W$ decays as $W \rightarrow l \nu(l=e, \mu)$ while the other as $W \rightarrow 2 j$ ( $j$ denotes a jet from a light quark). The branching fraction for this channel is $\operatorname{Br}(W W) \approx 2 / 9 \times 6 / 9 \times 2=8 / 27$ where the factor 2 is from exchanging the leptonic and hadronic decaying $W$ 's.

The signal of this channel is therefore $l+5 j$ with large missing transverse momentum carried away by a neutrino. The leading background is

$$
p p \rightarrow t \bar{t}+j \rightarrow l \nu+5 j
$$

The other background $p p \rightarrow W^{+} W^{-}+3 j$ is much smaller, not only because $W^{+} W^{-}$cross-section is smaller than $t \bar{t}$ cross-section but also because the two more QCD jets in this background introduce another factor of $\alpha_{s}^{2}$ suppression. We will thus only focus on the background of $t \bar{t}+1$ QCD jet.

We adopt the event selection criteria with the basic cuts [34]

$$
\begin{aligned}
& P_{T}(l)>25 \mathrm{GeV}, \quad|\eta(l)|<2.5, \\
& P_{T}(j)>20 \mathrm{GeV}, \quad|\eta(j)|<3, \\
& E_{T}>25 \mathrm{GeV}, \quad \Delta R_{j j, l j}>0.4 .
\end{aligned}
$$

We smear the lepton and jet energy approximately according to

$$
\delta E / E=\frac{a}{\sqrt{E / \mathrm{GeV}}} \oplus b
$$

where $a_{l}=13.4 \%, b_{l}=2 \%$ and $a_{j}=75 \%, b_{j}=3 \%$, and $\oplus$ denotes a sum in quadrature [35]. As shown in Table 3, the background is much higher than the signal if only applying the basic acceptance cuts. However, the signal has very unique kinematical features that we will utilize next to suppress the background and to reconstruct the signal.

One of striking features of the signal is that the $b$ jet from $W_{C}$ decay is very energetic due to the heavy mass of $W_{C}$. Among all the jets, this $b$ jet has the highest $P_{T}$ in most cases. Therefore, one can select the highest jet $P_{T}$ and impose cut on it

$$
P_{T}^{\text {highest }}>500 \mathrm{GeV}
$$

\footnotetext{
${ }^{20}$ We take the branching fractions of $\operatorname{Br}\left(t^{(1)} \rightarrow b W\right)$ as $50 \%$ in this estimate. This is a general feature for large $M_{t^{(1)}}$ only. It happens to be approximately true for the parameter point we use for this detailed study, although it corresponds to a small $M_{t(1)}$.
} 
Table 3: The cross sections (in fb) for the signal process $p p \rightarrow W_{C} t^{(1)} \rightarrow l \nu+5 j$ and SM background $p p \rightarrow t \bar{t}+j \rightarrow l \nu+5 j$, with the cuts and veto applied consecutively. Basic cuts refer to those in Eq. (53). The " $M_{3 j, l \nu j}$ cuts" refers to the cut condition in Eqs. (57) and (58), and " $M_{3 j}$ veto" refers to the veto condition in Eq. (59).

\begin{tabular}{|c|c|c|c|c|c|}
\hline & basic cuts & $P_{T}^{\text {highest }}$ & $E_{T}^{\text {vis }}$ & $M_{3 j, l \nu j}$ cuts & $M_{3 j}$ veto \\
\hline Signal & 0.045 & 0.040 & 0.037 & 0.025 & 0.025 \\
\hline Background & $2.4 \times 10^{4}$ & 76 & 8.7 & 1.7 & $<10^{-4}$ \\
\hline
\end{tabular}

Since the effective c.m. energy is quite large in signal for the heavy particle production, which are in general higher than those in background, we further impose cut on the scalar sum of the visible transverse energies of all the jets and $l$

$$
E_{T}^{v i s}>1.5 \mathrm{TeV}
$$

With the jet of highest $P_{T}$ identified as the $b$ jet from $W_{C}$ decay, there are 4 jets remaining. One can identify which three of them are from $t^{(1)}$ hadronic decay by selecting 3 jets which give the invariant mass closest to $t^{(1)}$, and require it to satisfy

$$
\left|M_{3 j}-M_{t^{(1)}}\right|<50 \mathrm{GeV},
$$

where, as discussed earlier, we have assumed that the mass of $t^{(1)}$ is known from the early search. Furthermore, the neutrino momentum can be fully reconstructed using $W$ mass condition $M_{W}^{2}=$ $\left(p_{l}+p_{\nu}\right)^{2}$ with a two-fold uncertainty 21. We select the solution which, in combination with the momenta of $l$ and the other remaining jet, gives the mass closer to $M_{t^{(1)}}$, and further require it to satisfy 22

$$
\left|M_{l \nu j}-M_{t^{(1)}}\right|<100 \mathrm{GeV} .
$$

With these done, the momenta of two $t^{(1)}$ 's are reconstructed. One still do not know which $t^{(1)}$ is from $W_{C}$ decay, and should try both of them, in combination with the jet with highest $P_{T}$, to reconstruct the $W_{C}$ mass. Since one of the two is expected to be the right one, the collection of the events should point to $M_{W_{C}}$ in the mass distribution.

Although this reconstruction procedure is highly efficient, with a signal efficiency about $56 \%$ and the background rejection of a factor of $10^{-4}$, as seen from Table 3, it is still not sufficient to

\footnotetext{
${ }^{21}$ In doing this, the neutrino $P_{T}$ is fixed to balance the $P_{T}$ of $l$ and jets, which have uncertainties due to smearing. To accommodate this uncertainty, we allow the $M_{W}$ to be as large as $120 \mathrm{GeV}$. It turns out that, with this range of $M_{W}$, there are still cases where there exist no solution for neutrino momentum, and we lose about $1 / 3$ of events in solving for the neutrino momentum.

${ }^{22}$ Here we assume $M_{t(1)}$ is known. On the other hand, without assuming this, one can still fix it by requiring there are 3 jets and $(l, \nu, j)$ having close heavy masses $\left(\gg m_{t}\right)$ and identify this common mass scale as $M_{t}(1)$.
} 
remove the background. However, beside identifying the characteristics of the signal, we also notice the features of the background. One of the essential and obvious features of the background is that there are two top quarks in the event, one of which decays to 3 jets. One can thus require that there be no combination of 3 jets with invariant mass within the top mass window

$$
\left|M_{3 j}-m_{t}\right|>50 \mathrm{GeV}
$$

This veto condition is highly efficient on removing the background.

We show, in Table 3, the cross sections of signal and background, with the cuts and veto applied consecutively. Both signal and background are obtained with parton-level Monte Carlo simulations, with detector effects accounted for by the geometrical acceptance and the energy smearing as discussed earlier. We see that the background is essentially removed with the veto condition of Eq. (59) applied.

In fact, the veto on $M_{3 j}$ alone would be sufficient to bring this $t \bar{t} j$ background below the signal. Our signal reconstruction scheme is nevertheless effective to single out the signal from the other potential backgrounds and to obtain the necessary knowledge about the masses of the heavy particle produced.

\subsection{2 $t^{(1)} t^{(1)} \rightarrow b W, \operatorname{th}(t Z)$}

We now consider the case with one $t^{(1)}$ decaying to $b W$ and the other decaying to $t h$ or $t Z$. Since the signals of the final states are rather similar for these two cases, we discuss them together. The signal we are looking for is

$$
b g \rightarrow W_{C} t^{(1)} \rightarrow b+2 t^{(1)} \rightarrow b b W t h(Z) \rightarrow l \nu+7 j,
$$

whose branching fraction (summing over both $t^{(1)} \rightarrow t h$ and $t Z$ ) is a product of three factors

$$
2 \times B r\left(W_{C} \rightarrow b t^{(1)}\right) \times B r\left(t^{(1)} \rightarrow b W\right) \times B r\left(t^{(1)} \rightarrow t h, t Z\right) \approx 2 \times 90 \% \times(50 \%)^{2}=45 \%,
$$

where the factor of 2 is from exchanging the decay mode of two $t^{(1)}$ 's. We consider semileptonic decay of two $W$ 's, which gives branching fraction 8/27 as discussed earlier.

The dominant SM background for this channel is from the

$$
p p \rightarrow t \bar{t}+3 j \rightarrow l \nu+7 j .
$$

This can be considered as adding two more QCD jets to the background considered in section 5.3.1. Although we expect this analysis directly analogous to that in the previous session, one may not be able to effectively calculate this multiple parton final state. Instead, we will thus simply give an estimate on how this background is suppressed by various cuts based on what we learn from the 
Monte Carlo simulations in section 5.3.1. To assess the signal/background ratio for this channel, we compare both the signal and background with those in the channel $\left(t^{(1)} t^{(1)} \rightarrow b W, b W\right)$ in the previous section.

For signal, this channel has larger branching fraction than the one considered in section 5.3 .1 , However, since there are two more jets in the final state, there are fewer events surviving the cuts of $\Delta R_{j j, l j}>0.4$. As shown in Table 4 , the signal cross-section after basic cuts in this channel is very close to that in the channel $\left(t^{(1)} t^{(1)} \rightarrow b W, b W\right)$.

The cuts on $P_{T}^{\text {highest }}$ and $E_{T}^{v i s}$ are still applicable to this channel. Again, the jet with the highest $P_{T}$ is identified as the $b$ jet from $W_{C}$ decay. Among the remaining 6 jets, one can require that there be at least one pair of jets with invariant mass close to either $M_{h}$, which we assume to be $125 \mathrm{GeV}$, or $M_{Z}$

$$
\left|M_{2 j}-M_{h}\right|<15 \mathrm{GeV} \text { or }\left|M_{2 j}-M_{Z}\right|<15 \mathrm{GeV}
$$

The two jets from $h$ or $Z$ decay can be identified in this way 23 . The rest of the procedure in reconstructing two $t^{(1)}$ momenta is similar to that in the $\left(t^{(1)} t^{(1)} \rightarrow b W, b W\right)$ channel.

Among all the remaining 4 jets, we require there exists at least one combination of 3 jets and $(l, \nu, j)$, which satisfy

$$
\begin{aligned}
& \left|M_{3 j}-m_{t}\right|<50 \mathrm{GeV} \text { and }\left|M_{l \nu j}-M_{t^{(1)}}\right|<100 \mathrm{GeV} \\
& \text { or } \quad\left|M_{3 j}-M_{t^{(1)}}\right|<50 \mathrm{GeV} \text { and }\left|M_{l \nu j}-m_{t}\right|<100 \mathrm{GeV} .
\end{aligned}
$$

Then, one can combine 2 jets which falls into the $h$ or $Z$ mass region with the cluster of either 3 jets or $(l, \nu, j)$, whichever falls into the $M_{t}$ region, and require this 5 jets or $(l, \nu, 3 j)$ has mass close to $M_{t^{(1)}}$

$$
\left|M_{5 j}-M_{t^{(1)}}\right|<50 \mathrm{GeV} \text { or }\left|M_{l \nu 3 j}-M_{t^{(1)}}\right|<100 \mathrm{GeV} \text {. }
$$

At this stage, the momenta of two $t^{(1)}$ 's are reconstructed, and one can go further to reconstruct $W_{C}$ mass by trying both $t^{(1)}$ 's with the jet with the highest $P_{T}$. Again, the correlation among events should point to the correct $M_{W_{C}}$.

Since the top quark also appears in the signal of this channel, the simple veto on top mass (used in section 5.3.1) is not applicable here. However, the background in this channel is sufficiently reduced to be below the signal with the above cuts applied. This is because there are two more suppressions in this channel. First, the $t \bar{t}+3 j$ background is further suppressed by $\alpha_{s}^{2} \approx 10^{-2}$ compared to that of $t \bar{t}+1 j$ background of the $\left(t^{(1)} t^{(1)} \rightarrow b W, b W\right)$ channel due to the appearance of two more QCD jets. Second, the extra cut on $M_{2 j}$ in Eq. (63) also introduce another factor of suppression, which we estimate it to be a factor of $10^{-1}$.

\footnotetext{
${ }^{23}$ If there are more than one pair of jets satisfying this, one selects the pair whose invariant mass is closer to $M_{H}$ or $M_{Z}$.
} 
Table 4: The cross-sections (in fb) for the signal process $p p \rightarrow W_{C} t^{(1)} \rightarrow l \nu+7 j$ and SM background $p p \rightarrow t \bar{t}+3 j \rightarrow l \nu+7 j$, with the cuts applied successively. Basic cuts refer to those in Eq. (53). The cuts on $E_{T}^{v i s}$ and $P_{T}^{\text {highest }}$ refer to Eqs. (55) and (56). The " $M_{2 j}$ cut" refer to Eq. (63). The " $M_{3 j, l \nu j}, M_{5 j, l \nu 3 j}$ cuts" refer to Eqs. (75) and (76).

\begin{tabular}{|c|c|c|c|c|c|}
\hline & basic cuts & $P_{T}^{\text {highest }}$ & $E_{T}^{v i s}$ & $M_{2 j}$ cut & $M_{3 j, l \nu j}, M_{5 j, l \nu 3 j}$ cuts \\
\hline Signal & 0.041 & 0.037 & 0.035 & 0.033 & 0.011 \\
\hline Background & $2.4 \times 10^{2}$ & 0.76 & 0.087 & 0.0087 & $<0.001$ \\
\hline
\end{tabular}

The cross-sections of signal and background in this channel, with cuts applied successively, are summarized in Table 4. The cross-section of signal is based on our parton-level Monte Carlo simulation, and that of background is obtained from an estimate based on the cross section of the background in the $t^{(1)} t^{(1)} \rightarrow b W, b W$ channel in 5.3 .1 .

\subsection{3 $t^{(1)} t^{(1)} \rightarrow \operatorname{th}(t Z), \operatorname{th}(t Z)$}

Finally, we consider the channel with both $t^{(1)}$ decaying to $t h(t Z)$

$$
b g \rightarrow W_{C} t^{(1)} \rightarrow b+2 t^{(1)} \rightarrow b t h(Z) t h(Z) \rightarrow l \nu+9 j,
$$

whose branching fraction (summing over both $t^{(1)} \rightarrow t h$ and $t Z$ ) is a product of three factors

$$
\operatorname{Br}\left(W_{C} \rightarrow b t^{(1)}\right) \times B r\left(t^{(1)} \rightarrow t h, t Z\right)^{2} \approx 90 \% \times(50 \%)^{2}=22.5 \% .
$$

We will again use semileptonic decay of two $W$ 's whose branching fraction is $8 / 27$ as discussed earlier.

The dominant SM background for this channel is from the $p p \rightarrow t \bar{t}+5 j \rightarrow l \nu+9 j$. We will simply give an estimate on how this background is suppressed by various cuts based on what we learned from the study based on Monte Carlo simulations in section 5.3.1

The branching fraction of this channel is similar to the $\left(t^{(1)} t^{(1)} \rightarrow b W, b W\right)$ channel. However, with four more jets in the signal event, the cross-section is drastically reduced after imposing basic cuts that involve $\Delta R_{j j, l j}>0.4$.

On the other hand, the background is smaller by a factor of $\alpha_{s}^{4} \approx 10^{-4}$ than in the $\left(t^{(1)} t^{(1)} \rightarrow\right.$ $b W, b W)$ channel. Some of the cuts discussed previously are still applicable in this channel. They include the cuts on $E_{T}^{v i s}, P_{T}^{\text {highest }}$, and $M_{2 j}$ (we require that there are at least two pairs of jets that satisfy Eq. (63) in this channel).

Similarly to the other two channels, the two $t^{(1)}$ momenta can be reconstructed and the $W_{C}$ mass can be obtained from correlations among events. We skip the details of this procedure here 
Table 5: The cross-sections (in fb) for the signal process $p p \rightarrow W_{C} t^{(1)} \rightarrow l \nu+9 j$ and SM background $p p \rightarrow t \bar{t}+5 j \rightarrow l \nu+9 j$, with the cuts applied successively. Basic cuts refer to those in Eq. (53). The $P_{T}$ cuts on $P_{T}^{\text {highest }}$ and $E_{T}^{\text {vis }}$ follow Eqs. (55) and (56), and invariant mass cuts on $M_{2 j}, M_{3 j, l \nu j}$, and $M_{5 j, l \nu 3 j}$ follows Eqs. (63), (68) and (66).

\begin{tabular}{|c|c|c|c|c|c|}
\hline & basic cuts & $P_{T}^{\text {highest }}$ & $E_{T}^{\text {vis }}$ & two $M_{2 j}$ cuts & $M_{3 j, l \nu j}, M_{5 j, l \nu 3 j}$ cuts \\
\hline Signal & 0.0081 & 0.0070 & 0.0068 & 0.0064 & 0.0023 \\
\hline Background & 2.4 & 0.0076 & 0.00087 & $<8.7 \times 10^{-6}$ & $<1.0 \times 10^{-6}$ \\
\hline
\end{tabular}

since it should be clear from discussion in the previous sections. One should note that the cuts on 3 jets and $(l, \nu, j)$ invariant mass in Eq. (75) should be replaced by

$$
\left|M_{3 j}-m_{t}\right|<50 \mathrm{GeV} \text { and }\left|M_{l \nu j}-m_{t}\right|<100 \mathrm{GeV},
$$

since there are two top quarks.

We show, in Table 5, the signal and background with the cuts applied successively. Again, the cross-section of signal is based on our parton-level Monte Carlo simulation, and that of background is based on our estimate built upon section 5.3.1.

\subsubsection{Summarizing all channels}

According to the study of each channel presented above, the background in each channel can be sufficiently suppressed after imposing various cut and veto criteria. For reader's convenience, we reiterate and summarize the cuts condition of all three channels here again.

Common cuts for all three channels:

(a) Basic cuts as in Eq. (53);

(b) $P_{T}$ cuts: $P_{T}^{\text {highest }}>500 \mathrm{GeV}, E_{T}^{\text {vis }}>1.5 \mathrm{TeV}$;

Specific cuts in each channels:

(i) Channel I $\left(l+5 j+E_{T}\right)$

(c) $M_{3 j, l \nu j}$ cuts: with the jet with highest $P_{T}$ excluded, requiring there exists such a combination of 3 jets and $(l, \nu, j)$ satisfying the invariant mass cut

$$
\left|M_{3 j}-M_{t^{(1)}}\right|<50 \mathrm{GeV},\left|M_{l \nu j}-M_{t^{(1)}}\right|<100 \mathrm{GeV}
$$

(d) $M_{3 j}$ veto: requiring there exist no combination of 3 jets that has invariant mass close to top mass

$$
\left|M_{3 j}-m_{t}\right|>50 \mathrm{GeV}
$$


(ii) Channel II $\left(l+7 j+E_{T}\right)$

(c) $M_{2 j}$ cut: with the jet with highest $P_{T}$ excluded, requiring there exists at least one pair of jets with mass close to $h$ or $Z$

$$
\left|M_{2 j}-M_{h}\right|<15 \mathrm{GeV} \text { or }\left|M_{2 j}-M_{Z}\right|<15 \mathrm{GeV}
$$

(d) $M_{3 j, l \nu j}$ cuts: within the remaining 4 jets, requiring there exists at least one combination of 3 jets and $(l, \nu, j)$ that satisfy

$$
\begin{aligned}
& \left|M_{3 j}-m_{t}\right|<50 \mathrm{GeV} \text { and }\left|M_{l \nu j}-M_{t^{(1)}}\right|<100 \mathrm{GeV} \\
\text { or } \quad & \left|M_{3 j}-M_{t^{(1)}}\right|<50 \mathrm{GeV} \text { and }\left|M_{l \nu j}-m_{t}\right|<100 \mathrm{GeV} .
\end{aligned}
$$

(e) $M_{5 j, l \nu 3 j}$ cuts: combining 2 jets that falls into the $h$ or $Z$ mass region with the cluster of either 3 jets or $(l, \nu, j)$, whichever falls into the $M_{t}$ region, and requiring this 5 jets or $(l, \nu, 3 j)$ has mass close to $M_{t^{(1)}}$

$$
\left|M_{5 j}-M_{t^{(1)}}\right|<50 \mathrm{GeV} \text { or }\left|M_{l \nu 3 j}-M_{t^{(1)}}\right|<100 \mathrm{GeV} \text {. }
$$

(iii) Channel III $\left(l+9 j+E_{T}\right)$

(c) $M_{2 j}$ cut: with the jet with highest $P_{T}$ excluded, requiring there exists at least two pairs of jets with mass close to $h$ or $Z$

$$
\left|M_{2 j}-M_{h}\right|<15 \mathrm{GeV} \text { or }\left|M_{2 j}-M_{Z}\right|<15 \mathrm{GeV}
$$

(d) $M_{3 j, l \nu j}$ cuts: within the remaining 4 jets, requiring there exists at least one combination of 3 jets and $(l, \nu, j)$ that satisfy

$$
\left|M_{3 j}-m_{t}\right|<50 \mathrm{GeV} \text { and }\left|M_{l \nu j}-m_{t}\right|<100 \mathrm{GeV} .
$$

(e) $M_{5 j, l \nu 3 j}$ cuts: combining 2 pairs of jets which falls into the $h$ or $Z$ mass region with 3 jets and $(l, \nu, j)$, and requiring that in one of the two possible combinations, both 5 jets and $(l, \nu, 3 j)$ have invariant mass satisfying

$$
\left|M_{5 j}-M_{t^{(1)}}\right|<50 \mathrm{GeV} \text { and }\left|M_{l \nu 3 j}-M_{t^{(1)}}\right|<100 \mathrm{GeV}
$$

To assess the discovery potential, we combine the number of events in all the channels based on the above study of the benchmark point in the parameter space (with $M_{W_{C}}=2 \mathrm{TeV}$ ). The luminosity needed at the $\mathrm{LHC}(14 \mathrm{TeV})$ for a $95 \%(2 \sigma), 99.7 \%(3 \sigma)$, and $99.9999 \%(5 \sigma)$ c.l. discovery of $W_{C}$, which implies about 3, 5, and 15 events, respectively (assuming that the background is 


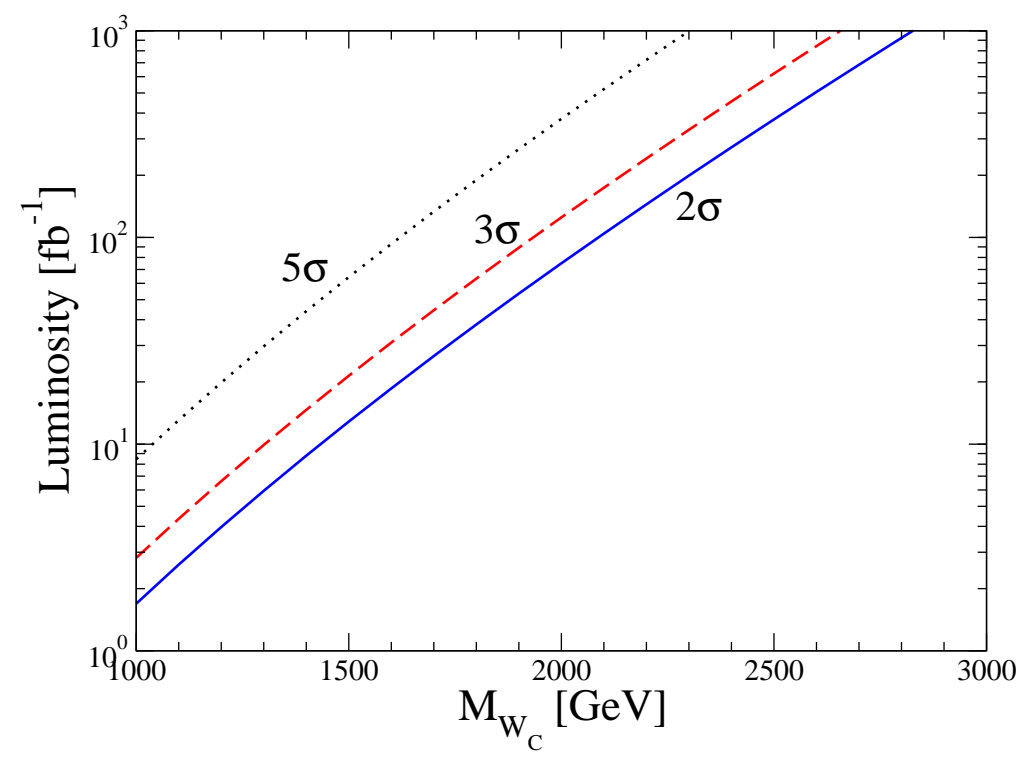

Figure 8: The luminosity needed for a $2 \sigma$ (blue solid line), $3 \sigma$ (red dashed line), and $5 \sigma$ (black dotted line) discovery of $W_{C}$ at $\mathrm{LHC}(14 \mathrm{TeV})$ as a function of $M_{W_{C}}$.

negligible, as is the case here), can then be determined. For other $M_{W_{C}}$ masses, we rescale this total number of events from the $2 \mathrm{TeV}$ case, based on the dependence of cross-section on $M_{W_{C}}$ as in Fig. 7. These results are displayed in Fig. 8. Conversely, for a luminosity of $1000 \mathrm{fb}^{-1}$, the reach in $M_{W_{C}}$ is $2.3 \mathrm{TeV}$ at $5 \sigma, 2.6 \mathrm{TeV}$ at $3 \sigma$, and $2.8 \mathrm{TeV}$ at $2 \sigma$.

\section{Discussions and Conclusions}

During the past decade, the framework of a warped extra dimension with the SM fields propagating in it has emerged as an attractive extension of the SM due to its potential for solving both the Planck-weak and flavor hierarchy problems of the SM. Moreover, naturalness motivates obtaining SM Higgs as a by-product of extending the $5 D$ EW gauge symmetry beyond the SM one and breaking it down to the SM near the $\mathrm{TeV}$ brane.

In this paper, we first give a full-fledged presentation for the formalism involving the coset gauge bosons in this framework, i.e., the extra (beyond SM-type) EW gauge bosons which are characteristically doublets under $\mathrm{SU}(2)_{L}$. We have then performed a study of LHC signals for the coset gauge boson $W_{C}$. We have developed a judicial and complex set of kinematical cuts to optimize the signal-to-background ratios. We have found that discovery of these gauge bosons at the LHC is very challenging. The primary reason for this is due to their unique gauge quantum 
numbers, so that the coset gauge bosons do not couple at leading order (in Higgs vev) to two SM particles, whether gauge bosons or fermions. Thus the $s$-channel resonant production of coset gauge bosons is quite suppressed, making production in association with KK top an important mechanism (assuming that the KK top can be as light $\sim 500 \mathrm{GeV}$, which does indeed happen quite commonly in this framework) and thus we focused on this channel in this paper. This associated production experiences phase space suppression.

On the other hand, the advantage of this feature of the couplings of coset gauge bosons is that their two-body decays to SM particles are also suppressed. The leading decay for charged coset gauge bosons is to $t^{(1)} b$, and in turn, $t^{(1)} \rightarrow b W$ or $t h, t Z$. Thus the final states are richer than just two SM particles, enabling separation from leading SM backgrounds, such as $t \bar{b}, t \bar{t}$. Based on such an analysis, we have estimated the $3 \sigma$ reach for coset gauge bosons to be $\sim 2(2.6) \mathrm{TeV}$ with $\sim 100$ (1000) $\mathrm{fb}^{-1}$ of luminosity. We notice, however, that typical models suggest that the mass scale of these gauge bosons is at least $\sim 3 \mathrm{TeV}$. This expectation is based on an indirect limit from precision EW observables (direct bound on coset gauge boson mass being weaker), namely, due to the masses of coset gauge boson and those of the KK excitations of SM gauge bosons being related and the latter being directly constrained by precision EW observables.

The same feature of the coset gauge boson couplings also makes their signals distinct from those of other heavy EW gauge bosons as follows. Consider the signals for the EW KK gauge bosons with the same quantum numbers as the SM gauge bosons within the same framework, i.e., KK $Z, W$ and $\gamma$ [30]. They couple at leading order to two SM particles, for example, to third generation quarks, $W W / W Z 24$ and even to two light quarks (but typically with suppressed couplings compared to the SM ones), unlike coset gauge bosons which do not couple to $W W / W Z$ or to two SM quarks at leading order in Higgs vev. Thus, the production cross-section is larger for $\mathrm{KK} W / Z / \gamma$ than for coset gauge bosons of the same mass, but the decay channels of $\mathrm{KK} W / Z$ are not as rich as for coset gauge bosons. We can also compare to signals for Little Higgs models, where $Z^{\prime} / W^{\prime}$, without $T$-parity, generically do couple to (and hence can be produced by or decay into) $W W / W Z$ or to two SM light fermions [36], resulting in distinct signatures from those of the coset gauge bosons. Similarly, $4 D$ Left-Right symmetric models have a $W_{R}^{+}$which does not couple to $W / Z$ at leading order but it does couple to two SM right-handed quarks and hence it can be produced by light quark-antiquark annihilation [37] and can decay into $t_{R} \bar{b}_{R}$. Finally, we must note that for many of the gauge sector extensions to include $Z^{\prime} / W^{\prime}$ (KK $W / Z$, photon in warped extra dimensional framework being notable exceptions), their leptonic decays are always the gold-plated signatures, which is absent for the coset gauge boson searches.

\footnotetext{
${ }^{24}$ Note that there are actually more than one neutral and charges states so that one mass eigenstate (i.e., admixture of gauge eigenstates) might have suppressed couplings due to cancelation between its various components, but then the other (almost degenerate) state does not have such suppressed couplings.
} 
We envisage the following sequence of events if this framework were realized in Nature: It is likely that a light KK top $t^{(1)}$ will be the first new particles to be discovered, with possibly less than $10 \mathrm{fb}^{-1}$ luminosity. With about $100 \mathrm{fb}^{-1}$ of integrated luminosity, it is the turn of the KK gluon next via its decay to $t \bar{t}$, followed closely by the $\mathrm{KK} W / Z$ via both gauge boson $W W / W Z$ and fermion $t \bar{b} / t \bar{t}$ final states 25 . Finally with even higher luminosity, the coset gauge bosons can be searched for using final states with top/bottom/ $W$ (i.e., like some decays of $\mathrm{KK} W / Z$, but with extra particles), but with no corresponding signal/excess in $W W / W Z$ final states. Although the signatures of the new particles in the warped extra dimensional framework (including those of coset gauge bosons in the more natural versions) are qualitatively distinctive, it is clear that their detailed analyses at the LHC would be required in order to establish this attractive theoretical framework.

\section{Acknowledgments}

We would like to thank Roberto Contino and Raman Sundrum for valuable discussions. K.A. was supported in part by an NSF grant No. PHY-0652363. T.H. was supported by the US DOE under contract DE-FG02-95ER40896. Y.L. was supported by the US DOE under contract DE-FG02$08 \mathrm{ER} 41531$.

\section{A $S O(5)$ generators and group algebras}

The commutation relations of $S O(5)$ generators are given by

$$
\begin{array}{r}
{\left[T_{L}^{a}, T_{L}^{b}\right]=i \epsilon^{a b c} T_{L}^{c}, \quad\left[T_{R}^{a}, T_{R}^{b}\right]=i \epsilon^{a b c} T_{R}^{c}, \quad\left[T_{L}^{a}, T_{R}^{b}\right]=0} \\
{\left[T^{\hat{a}}, T^{\hat{b}}\right]=\frac{i}{2} \epsilon^{a b c}\left(T_{L}^{c}+T_{R}^{c}\right), \quad\left[T^{\hat{a}}, T^{\hat{4}}\right]=\frac{i}{2}\left(T_{L}^{a}-T_{R}^{a}\right)} \\
{\left[T_{L, R}^{a}, T^{\hat{b}}\right]=\frac{i}{2}\left(\epsilon^{a b c} T^{\hat{c}} \pm \delta^{a b} T^{\hat{4}}\right), \quad\left[T_{L, R}^{a}, T^{\hat{4}}\right]=\mp \frac{i}{2} T^{\hat{a}}}
\end{array}
$$

\footnotetext{
${ }^{25}$ although KK gluon decays to $t \bar{t}$ could be a "background" for KK $Z$ in the $t \bar{t}$ channel.
} 
For 5 representation, the generators are

$$
\begin{aligned}
& T_{L, R}^{1}=\frac{-i}{2}\left(\begin{array}{ccccc}
0 & 0 & 0 & \pm 1 & 0 \\
0 & 0 & 1 & 0 & 0 \\
0 & -1 & 0 & 0 & 0 \\
\mp 1 & 0 & 0 & 0 & 0 \\
0 & 0 & 0 & 0 & 0
\end{array}\right), \quad T_{L, R}^{2}=\frac{-i}{2}\left(\begin{array}{ccccc}
0 & 0 & -1 & 0 & 0 \\
0 & 0 & 0 & \pm 1 & 0 \\
1 & 0 & 0 & 0 & 0 \\
0 & \mp 1 & 0 & 0 & 0 \\
0 & 0 & 0 & 0 & 0
\end{array}\right) \\
& T_{L, R}^{3}=\frac{-i}{2}\left(\begin{array}{ccccc}
0 & 1 & 0 & 0 & 0 \\
-1 & 0 & 0 & 0 & 0 \\
0 & 0 & 0 & \pm 1 & 0 \\
0 & 0 & \mp 1 & 0 & 0 \\
0 & 0 & 0 & 0 & 0
\end{array}\right), \quad T^{\hat{1}}=\frac{-i}{\sqrt{2}}\left(\begin{array}{ccccc}
0 & 0 & 0 & 0 & 1 \\
0 & 0 & 0 & 0 & 0 \\
0 & 0 & 0 & 0 & 0 \\
0 & 0 & 0 & 0 & 0 \\
-1 & 0 & 0 & 0 & 0
\end{array}\right) \\
& T^{\hat{2}}=\frac{-i}{\sqrt{2}}\left(\begin{array}{ccccc}
0 & 0 & 0 & 0 & 0 \\
0 & 0 & 0 & 0 & 1 \\
0 & 0 & 0 & 0 & 0 \\
0 & 0 & 0 & 0 & 0 \\
0 & -1 & 0 & 0 & 0
\end{array}\right), \quad T^{\hat{3}}=\frac{-i}{\sqrt{2}}\left(\begin{array}{ccccc}
0 & 0 & 0 & 0 & 0 \\
0 & 0 & 0 & 0 & 0 \\
0 & 0 & 0 & 0 & 1 \\
0 & 0 & 0 & 0 & 0 \\
0 & 0 & -1 & 0 & 0
\end{array}\right) \\
& T^{\hat{4}}=\frac{-i}{\sqrt{2}}\left(\begin{array}{ccccc}
0 & 0 & 0 & 0 & 0 \\
0 & 0 & 0 & 0 & 0 \\
0 & 0 & 0 & 0 & 0 \\
0 & 0 & 0 & 0 & 1 \\
0 & 0 & 0 & -1 & 0
\end{array}\right)
\end{aligned}
$$

\section{B KK Decomposition and Spectral Functions of Gauge Bosons and Fermions}

In this appendix, we review the KK decomposition and spectral functions of gauge bosons and fermions in the model[ 8 . First, we define base functions for gauge bosons

$$
\begin{aligned}
C_{A}\left(z, m_{n}\right) & =\frac{\pi m_{n}}{2} z\left[J_{1}\left(m_{n} z\right) Y_{0}\left(m_{n}\right)-J_{0}\left(m_{n}\right) Y_{1}\left(m_{n} z\right)\right] \\
S_{A}\left(z, m_{n}\right) & =\frac{\pi m_{n}}{2} z\left[J_{1}\left(m_{n}\right) Y_{1}\left(m_{n} z\right)-J_{1}\left(m_{n} z\right) Y_{1}\left(m_{n}\right)\right]
\end{aligned}
$$

These base functions are like cosine and sine in flat extra dimension. $C_{A}\left(z, m_{n}\right)$ has "+" boundary condition on the UV brane and $S_{A}\left(z, m_{n}\right)$ has "-" boundary condition on the UV brane. They both solve the equations of motion for gauge boson wavefunctions with vanishing Higgs vev. Then the wavefunctions of gauge fields with vanishing Higgs vev are easy to write down in terms of these base functions

$$
\begin{array}{r}
f_{n}^{a_{L}}(z, 0)=C_{a_{L}, n} C_{A}\left(z, m_{n}\right) \\
f_{n}^{\hat{a}}(z, 0)=C_{\hat{a}, n} S_{A}\left(z, m_{n}\right) \\
f_{n}^{Y}(z, 0)=C_{Y, n} C_{A}\left(z, m_{n}\right) \\
f_{n}^{a_{R}}(z, 0)=C_{a_{R}, n} S_{A}\left(z, m_{n}\right)
\end{array}
$$


The wavefunctions in the presence of background $\left\langle A_{z}^{\hat{4}}\right\rangle$ can be obtained by using Eq. (28). For simplicity, we define

$$
\theta_{G}(z)=\frac{v\left(z^{2}-R^{2}\right)}{\sqrt{2} f_{h}\left(z_{\pi}^{2}-R^{\prime 2}\right)}
$$

Then $\Omega(z, v)=e^{-i \sqrt{2} \theta_{G}(z) T^{\hat{4}}}$. Use the representation of $S O(5)$ generators in Appendix $\mathrm{A}$, we obtain

$$
\begin{aligned}
f^{1_{L}}(v) & =\frac{1}{2}\left(1+\cos \theta_{G}\right) C_{1_{L}} C_{A}(z)+\frac{1}{2}\left(1-\cos \theta_{G}\right) C_{1_{R}} S_{A}(z)+\frac{\sqrt{2}}{2} \sin \theta_{G} C_{\hat{1}} S_{A}(z) \\
f^{2_{L}}(v) & =\frac{1}{2}\left(1+\cos \theta_{G}\right) C_{2_{L}} C_{A}(z)+\frac{1}{2}\left(1-\cos \theta_{G}\right) C_{2_{R}} S_{A}(z)+\frac{\sqrt{2}}{2} \sin \theta_{G} C_{\hat{2}} S_{A}(z) \\
f^{3_{L}}(v) & =\frac{1}{2}\left(1+\cos \theta_{G}\right) C_{3_{L}} C_{A}(z)+\frac{1}{2}\left(1-\cos \theta_{G}\right)\left[c_{\phi} C_{3_{R}} S_{A}(z)+s_{\phi} C_{Y} C_{A}(z)\right] \\
& +\frac{\sqrt{2}}{2} \sin \theta_{G} C_{\hat{3}} S_{A}(z) \\
f^{1_{R}}(v) & =\frac{1}{2}\left(1-\cos \theta_{G}\right) C_{1_{L}} C_{A}(z)+\frac{1}{2}\left(1+\cos \theta_{G}\right) C_{1_{R}} S_{A}(z)-\frac{\sqrt{2}}{2} \sin \theta_{G} C_{\hat{1}} S_{A}(z) \\
f^{2_{R}}(v) & =\frac{1}{2}\left(1-\cos \theta_{G}\right) C_{2_{L}} C_{A}(z)+\frac{1}{2}\left(1+\cos \theta_{G}\right) C_{2_{R}} S_{A}(z)-\frac{\sqrt{2}}{2} \sin \theta_{G} C_{\hat{2}} S_{A}(z) \\
f^{3_{R}}(v) & =\frac{1}{2}\left(1-\cos \theta_{G}\right) C_{3_{L}} C_{A}(z)+\frac{1}{2}\left(1+\cos \theta_{G}\right)\left[c_{\phi} C_{3_{R}} S_{A}(z)+s_{\phi} C_{Y} C_{A}(z)\right] \\
& -\frac{\sqrt{2}}{2} \sin \theta_{G} C_{\hat{3}} S_{A}(z) \\
f^{\hat{1}}(v) & =\cos \theta_{G} C_{\hat{1}} S_{A}(z)+\sin \theta_{G} \frac{1}{\sqrt{2}}\left[C_{1_{R}} S_{A}(z)-C_{1_{L}} C_{A}(z)\right] \\
f^{\hat{2}}(v) & =\cos \theta_{G} C_{\hat{2}} S_{A}(z)+\sin \theta_{G} \frac{1}{\sqrt{2}}\left[C_{2_{R}} S_{A}(z)-C_{2_{L}} C_{A}(z)\right] \\
f^{\hat{3}}(v) & =\cos \theta_{G} C_{\hat{3}} S_{A}(z)+\sin \theta_{G} \frac{1}{\sqrt{2}}\left[c_{\phi} C_{3_{R}} S_{A}(z)+s_{\phi} C_{Y} C_{A}(z)-C_{3_{L}} C_{A}(z)\right] \\
f^{\hat{4}}(v) & =C_{\hat{4}} S_{A}(z) \\
f^{X}(v) & =c_{\phi} C_{Y} C_{A}(z)-s_{\phi} C_{3_{R}} S_{A}(z)
\end{aligned}
$$

where the dependence on $z$ and KK number $n$ is not shown explicitly. The boundary conditions of $f^{a, \hat{a}}(v, z)$ at $z=R^{\prime}$ set the eigenvalues $m_{n}$. We can separate the gauge bosons in three sectors: (i) $a=1_{L}, 1_{R}, \hat{1}, 2_{L}, 2_{R}, \hat{2}$, these gauge bosons correspond to $W^{ \pm}$and their $\mathrm{KK}$ modes and coset $W_{C}$ gauge boson. (ii) $a=3_{L}, 3_{R}, \hat{3}, X$, these correspond to neutral gauge bosons $(Z, \gamma$ and coset $Z_{C}$ gauge boson). (iii) $a=\hat{4}$, corresponds to the gauge boson partner of physical Higgs boson. We now study these sectors.

- (i) $W^{ \pm}$sector. The boundary conditions on the IR brane are

$$
\begin{aligned}
\partial_{z} f_{i_{L}}\left(z_{\pi}, v\right) & =0 \\
\partial_{z} f_{i_{R}}\left(z_{\pi}, v\right) & =0 \\
f_{\hat{i}}\left(z_{\pi}, v\right) & =0
\end{aligned}
$$


where $i=1,2$. These boundary conditions give us

$$
\begin{aligned}
& C_{i_{L}}\left[C_{A}\left(R^{\prime}\right)-\sin \theta_{G} C_{A}\left(z_{\pi}\right) \theta_{G}^{\prime}+\cos \theta_{G} C_{A}^{\prime}\left(R^{\prime}\right)\right] \\
+ & C_{i_{R}}\left[S_{A}^{\prime}\left(R^{\prime}\right)+\sin \theta_{G} S_{A}\left(R^{\prime}\right) \theta_{G}^{\prime}-\cos \theta_{G} S_{A}^{\prime}\left(R^{\prime}\right)\right] \\
+ & C_{\hat{i}}\left[\sqrt{2} \cos \theta_{G} \theta_{G}^{\prime} S_{A}\left(R^{\prime}\right)+\sqrt{2} \sin \theta_{G} S_{A}^{\prime}\left(R^{\prime}\right)\right]=0 \\
& C_{i_{L}}\left[C_{A}^{\prime}\left(R^{\prime}\right)+\sin \theta_{G} C_{A}\left(R^{\prime}\right) \theta_{G}^{\prime}-\cos \theta_{G} C_{A}^{\prime}\left(R^{\prime}\right)\right] \\
+ & C_{i_{R}}\left[S_{A}^{\prime}\left(R^{\prime}\right)-\sin \theta_{G} S_{A}\left(R^{\prime}\right) \theta_{G}^{\prime}+\cos \theta_{G} S_{A}^{\prime}\left(R^{\prime}\right)\right] \\
- & C_{\hat{i}}\left[\sqrt{2} \cos \theta_{G} \theta_{G}^{\prime} S_{A}\left(R^{\prime}\right)+\sqrt{2} \sin \theta_{G} S_{A}^{\prime}\left(R^{\prime}\right)\right]=0 \\
& \cos \theta_{G} C_{\hat{i}} S_{A}\left(R^{\prime}\right)+\sin \theta_{G} \frac{1}{\sqrt{2}}\left[C_{i_{R}} S_{A}\left(R^{\prime}\right)-C_{i_{L}} C_{A}\left(R^{\prime}\right)\right]=0
\end{aligned}
$$

where all functions are evaluated at $z=R^{\prime}$. These are linear algebraic equations for the coefficients $C_{i_{L}, i_{R}, \hat{i}}$. To have a solution on the coefficients $C_{i_{L}, i_{R}, \hat{i}}$, we need to require the determinant to be zero. It gives us

$$
C_{A}\left(R^{\prime}\right) S_{A}^{\prime}\left(R^{\prime}\right) \sin ^{2} \theta_{G}+\left(2-\sin ^{2} \theta_{G}\right) C_{A}^{\prime}\left(R^{\prime}\right) S_{A}\left(R^{\prime}\right)=0
$$

which can be further simplified to

$$
\begin{gathered}
1+F_{W}\left(m_{n}^{2}\right) \sin ^{2}\left(\frac{v}{\sqrt{2} f_{h}}\right)=0 \\
F_{W}\left(m^{2}\right) \equiv \frac{m R^{\prime}}{2 C^{\prime}\left(R^{\prime}, m\right) S\left(R^{\prime}, m\right)}
\end{gathered}
$$

Here we defined the form factor of $\mathrm{W}$ bosons $F_{W}\left(m^{2}\right)$. Now we can see that the spectral function of $W$ boson is

$$
\rho_{W}(m)=1+F_{W}\left(m^{2}\right) \sin ^{2}\left(\frac{v}{\sqrt{2} f_{h}}\right)
$$

- (ii) $Z, \gamma$ sector. The boundary conditions on the IR brane are

$$
\begin{aligned}
\partial_{z} f_{3_{L}}\left(z_{\pi}, v\right) & =0 \\
\partial_{z}\left[c_{\phi} f_{3_{R}}\left(z_{\pi}, v\right)-s_{\phi} f_{X}\left(z_{\pi}, v\right)\right] & =0 \\
\partial_{z}\left[s_{\phi} f_{3_{R}}\left(z_{\pi}, v\right)+c_{\phi} f_{X}\left(z_{\pi}, v\right)\right] & =0 \\
f_{\hat{3}}\left(z_{\pi}, v\right) & =0
\end{aligned}
$$


These boundary conditions give us

$$
\begin{aligned}
& C_{3_{L}}\left[C_{A}^{\prime}\left(R^{\prime}\right)-\sin \theta_{G} C_{A}\left(R^{\prime}\right) \theta_{G}^{\prime}+\cos \theta_{G} C_{A}^{\prime}\left(R^{\prime}\right)\right] \\
+ & C_{3_{R}} c_{\phi}\left[S_{A}^{\prime}\left(R^{\prime}\right)+\sin \theta_{G} S\left(R^{\prime}\right) \theta_{G}^{\prime}-\cos \theta_{G} S_{A}^{\prime}\left(R^{\prime}\right)\right] \\
+ & C_{Y} s_{\phi}\left[C_{A}^{\prime}\left(R^{\prime}\right)+\sin \theta_{G} C_{A}\left(R^{\prime}\right) \theta_{G}^{\prime}-\cos \theta_{G} C_{A}^{\prime}\left(R^{\prime}\right)\right] \\
+ & \sqrt{2} C_{\hat{3}}\left[\sin \theta_{G} S_{A}^{\prime}\left(R^{\prime}\right)+\cos \theta_{G} S_{A}\left(R^{\prime}\right) \theta_{G}^{\prime}\right]=0 \\
& C_{3_{L}} c_{\phi}\left[C_{A}^{\prime}\left(R^{\prime}\right)-\cos \theta_{G} C_{A}^{\prime}\left(R^{\prime}\right)+\sin \theta_{G} C_{A}\left(R^{\prime}\right) \theta_{G}^{\prime}\right] \\
+ & C_{3_{R}}\left[\left(1+s_{\phi}^{2}\right) S_{A}^{\prime}\left(R^{\prime}\right)+c_{\phi}^{2} \cos \theta_{G} S_{A}^{\prime}\left(R^{\prime}\right)-c_{\phi}^{2} \sin \theta_{G} S_{A}\left(R^{\prime}\right) \theta_{G}^{\prime}\right] \\
+ & C_{Y} s_{\phi} c_{\phi}\left[-C_{A}^{\prime}\left(R^{\prime}\right)-\sin \theta_{G} C_{A}\left(R^{\prime}\right) \theta_{G}^{\prime}+\cos \theta_{G} C_{A}^{\prime}\left(R^{\prime}\right)\right] \\
- & C_{\hat{3}} \sqrt{2} c_{\phi}\left[\sin \theta_{G} S_{A}^{\prime}\left(R^{\prime}\right)+\cos \theta_{G} S_{A}\left(R^{\prime}\right) \theta_{G}^{\prime}\right]=0 \\
& C_{3_{L}} s_{\phi}\left[C_{A}^{\prime}\left(R^{\prime}\right)-\cos \theta_{G} C_{A}^{\prime}\left(R^{\prime}\right)+\sin \theta_{G} C_{A}\left(R^{\prime}\right) \theta_{G}^{\prime}\right] \\
+ & C_{3_{R}} s_{\phi} c_{\phi}\left[-S_{A}^{\prime}\left(R^{\prime}\right)+\cos \theta_{G} S_{A}^{\prime}\left(R^{\prime}\right)-\sin \theta_{G} S_{A}\left(R^{\prime}\right) \theta_{G}^{\prime}\right] \\
+ & C_{Y}\left[\left(1+c_{\phi}^{2}\right) C_{A}^{\prime}\left(R^{\prime}\right)+s_{\phi}^{2} \cos \theta_{G} C_{A}^{\prime}\left(R^{\prime}\right)-s_{\phi}^{2} \sin \theta_{G} C_{A}\left(R^{\prime}\right) \theta_{G}^{\prime}\right] \\
- & C_{\hat{3}} \sqrt{2} s_{\phi}\left[\sin \theta_{G} S_{A}^{\prime}\left(R^{\prime}\right)+\cos \theta_{G} S_{A}\left(R^{\prime}\right) \theta_{G}^{\prime}\right]=0 \\
& \\
& C_{3_{L}}\left[-\sin \theta_{G} C_{A}\left(R^{\prime}\right)\right]+C_{3_{R}}\left[c_{\phi} \sin \theta_{G} S_{A}\left(R^{\prime}\right)\right] \\
+ & C_{Y}\left[s_{\phi} \sin \theta_{G} C_{A}\left(R^{\prime}\right)\right]+C_{\hat{3}}\left[\sqrt{2} \cos \theta_{G} S_{A}\left(R^{\prime}\right)\right]=0
\end{aligned}
$$

By requiring the determinant is zero, we get

$$
C_{A}^{\prime}\left(R^{\prime}\right) S_{A}^{\prime}\left(R^{\prime}\right)\left\{2 C_{A}^{\prime}\left(R^{\prime}\right) S_{A}\left(R^{\prime}\right)+\sin ^{2} \theta_{G}\left(1+s_{\phi}^{2}\right)\left[C_{A}^{\prime}\left(R^{\prime}\right) S_{A}\left(R^{\prime}\right)-C_{A}\left(R^{\prime}\right) S_{A}^{\prime}\left(R^{\prime}\right)\right]\right\}=0(
$$

$C_{A}^{\prime}\left(R^{\prime}\right)=0$ gives the spectrum of $\mathrm{KK}$ photon and $S_{A}^{\prime}\left(R^{\prime}\right)=0$ gives the spectrum of KK $W_{3_{R}}$. Note that their spectrum does not depend on the Higgs vev thus does not contribute to the $\mathrm{CW}$ potential. With some simplification we can get the spectral function for $Z$ boson

$$
\rho_{Z}(m)=1+F_{Z}\left(m^{2}\right) \sin ^{2}\left(\frac{v}{\sqrt{2} f_{h}}\right)
$$

with the $Z$ boson form factor

$$
F_{Z}\left(m^{2}\right)=\frac{\left(1+s_{\phi}^{2}\right) m R^{\prime}}{2 C^{\prime}\left(R^{\prime}, m\right) S\left(R^{\prime}, m\right)}
$$

- (iii) $A^{\hat{4}}$ sector. The gauge transformation $\Omega(z, v)$ does not change the wavefunction of $A^{\hat{4}}$. Therefore

$$
f_{n}^{\hat{4}}(z, v)=f_{n}^{\hat{4}}(z, 0)=C_{\hat{4}, n} S_{A}\left(z, m_{n}\right)
$$


Its spectrum is determined by $S_{A}\left(R^{\prime}, m_{n}\right)=0$. Since the spectral function does not depend on the Higgs vev, it will not contribute to the Higgs potential.

For the fermionic section, we define the following base function

$$
\begin{aligned}
\tilde{S}_{M}^{F}\left(z, m_{n}\right) & =\frac{\pi m_{n}}{2} z^{\alpha}\left[J_{\alpha}\left(m_{n}\right) Y_{\alpha}\left(m_{n} z\right)-Y_{\alpha}\left(m_{n}\right) J_{\alpha}\left(m_{n} z\right)\right] \\
S_{ \pm M}^{F} & =z^{2 \pm M} \tilde{S}_{ \pm M}^{F} \\
\dot{S}_{ \pm M}^{F} & =\mp \frac{z^{2 \pm M}}{m_{n}} \partial_{z} \tilde{S}_{ \pm M}^{F}
\end{aligned}
$$

with $\alpha=1 / 2+c$ and $M=-c . \quad S_{ \pm M}$ and $\dot{S}_{ \pm M}$ satisfy Dirichlet and Neunman boundary conditions respectively at the UV brane. We can do the following KK decomposition for fermionic wavefunctions with vanishing Higgs vev

$$
F_{1 L}^{\Psi}(z, 0)=\left(\begin{array}{c}
C_{1} S_{M_{1}}^{F} \\
C_{2} S_{M_{1}}^{F} \\
C_{3} \dot{S}_{-M_{1}}^{F} \\
C_{4} \dot{S}_{-M_{1}}^{F} \\
C_{5} S_{M_{1}}^{F}
\end{array}\right), \quad F_{2 R}^{\Psi}(z, 0)=\left(\begin{array}{c}
C_{6} S_{-M_{2}}^{F} \\
C_{7} S_{-M_{2}}^{F} \\
C_{8} S_{-M_{2}}^{F} \\
C_{9} \dot{S}_{-M_{2}}^{F} \\
C_{10} \dot{S}_{M_{2}}^{F}
\end{array}\right), \quad F_{3 R}^{\Psi}(z, 0)=\left(\begin{array}{c}
C_{11} S_{-M_{3}}^{F} \\
C_{12} S_{-M_{3}}^{F} \\
C_{13} S_{-M_{3}}^{F} \\
C_{14} S_{-M_{3}}^{F} \\
C_{15} S_{-M_{3}}^{F} \\
C_{16} S_{-M_{3}}^{F} \\
C_{17} S_{-M_{3}}^{F} \\
C_{18} S_{-M_{3}}^{F} \\
C_{19} S_{-M_{3}}^{F} \\
C_{20} \dot{S}_{M_{3}}^{F}
\end{array}\right)
$$

As before, the wavefunctions with non-vanishing Higgs vev is given by doing gauge transformation Eq. (30). The boundary terms in Eq. (26) give twisted boundary conditions for fermions at the IR brane

$$
\begin{aligned}
& \chi_{1 R}+M_{B_{2}} \chi_{3 R}=0, \quad \tilde{t}_{1 R}+M_{B_{2}} \tilde{t}_{3 R}=0, \quad t_{1 R}+M_{B_{2}} t_{3 R}=0 \\
& b_{1 R}+M_{B_{2}} b_{3 R}=0, \quad \hat{t}_{1 R}+M_{B_{1}} \hat{t}_{2 R}=0, \quad \chi_{3 L}-M_{B_{2}} \chi_{1 L}=0 \\
& \tilde{t}_{3 L}-M_{B_{2}} \tilde{t}_{1 L}=0, \quad t_{3 L}-M_{B_{2}} t_{1 L}=0, \quad b_{3 L}-M_{B_{2}} b_{1 L}=0, \quad \hat{t}_{2 L}-M_{B_{1}} \hat{t}_{1 L}=0
\end{aligned}
$$

The rest of the boundary conditions are not changed

$$
\begin{array}{r}
\left(\chi_{2 L}, \hat{t}_{2 L}, t_{2 L}, b_{2 L}\right)=0 \\
\left(\Xi_{3 L}^{\prime}, T_{3 L}^{\prime}, B_{3 L}^{\prime}, \Xi_{3 L}, T_{3 L}, B_{3 L}\right)=0
\end{array}
$$

This boundary conditions set the mass spectra for fermions. The calculation for fermionic spectral function is similar to the case of gauge boson. We do not carry out the calculation here but present the fermionic form factors and spectral functions here for reference (for more detailed calculation, 
see [8]). The fermionic form factors are

$$
\begin{aligned}
F_{b}\left(m^{2}\right) & =-\frac{M_{B_{2}}^{2} S_{-c_{1}}^{F^{\prime}}}{2 S_{c_{3}}^{F}\left(M_{B_{2}}^{2} S_{-c_{3}}^{F} S_{-c_{1}}^{F \prime}+S_{-c_{1}}^{F} S_{-c_{3}}^{F \prime}\right)} \\
F_{t_{1}}\left(m^{2}\right) & =\frac{F_{1}\left(m^{2}\right)}{F_{0}\left(m^{2}\right)} \\
F_{t_{2}}\left(m^{2}\right) & =\frac{F_{2}\left(m^{2}\right)}{F_{0}\left(m^{2}\right)} \\
F_{1}\left(m^{2}\right) & =k z\left\{M_{B_{2}}^{2} S_{c_{2}}^{F} S_{-c_{3}}^{F} S_{-c_{2}}^{F \prime}+M_{B_{1}}^{2}\left[2 M_{B_{2}}^{2} S_{c_{1}}^{F} S_{-c_{3}}^{F} S_{-c_{1}}^{F \prime}\right.\right. \\
& \left.\left.+S_{-c_{3}}^{F \prime}+2 S_{c_{1}}^{F} S_{-c_{1}}^{F} S_{-c_{3}}^{F \prime}-S_{c_{2}}^{F} S_{-c_{2}}^{F} S_{-c_{3}}^{F \prime}\right]\right\} \\
F_{2}\left(m^{2}\right) & =-(k z) M_{B_{1}}^{2} S_{-c_{3}}^{F \prime} \\
F_{0}\left(m^{2}\right) & =2\left\{M_{B_{1}}^{2} S_{c_{1}}^{F}\left(-1+S_{c_{2}}^{F} S_{-c_{2}}^{F}\right)\left(M_{B_{2}}^{2} S_{-c_{3}}^{F} S_{-c_{1}}^{F \prime} k z+S_{-c_{1}}^{F} S_{-c_{3}}^{F \prime} k z\right)\right. \\
& \left.+S_{c_{2}}^{F} S_{-c_{2}}^{F \prime} k z\left[M_{B_{2}}^{2}\left(-1+S_{c_{1}}^{F} S_{-c_{1}}^{F}\right) S_{-c_{3}}^{F}-\frac{1}{m^{2}} S_{-c_{1}}^{F} S_{c_{1}}^{F \prime} S_{-c_{3}}^{F \prime}\right]\right\}
\end{aligned}
$$

The fermionic spectral functions are given by

$$
\begin{aligned}
& \rho_{b}(m)=1+F_{b}\left(m^{2}\right) \sin ^{2}\left(\frac{v}{\sqrt{2} f_{h}}\right), \\
& \rho_{t}(m)=1+F_{t_{1}}\left(m^{2}\right) \sin ^{2}\left(\frac{v}{\sqrt{2} f_{h}}\right)+F_{t_{2}}\left(m^{2}\right) \sin ^{4}\left(\frac{v}{\sqrt{2} f_{h}}\right) .
\end{aligned}
$$

\section{Suppression of $Z_{c} b_{L} \bar{b}_{L}$ coupling}

In the main text we commented that the couplings of the SM $b$ quark to the coset $Z_{C}$ gauge boson are strongly suppressed compared to the their naive estimates. In this appendix we will explain the origin of this suppression. From isospin quantum numbers of the coset gauge bosons $\left(T_{L}^{3}, T_{R}^{3}\right)=\left( \pm \frac{1}{2}, \pm \frac{1}{2}\right)$, we see that to get coupling between coset gauge boson and SM fermion we need odd number of Higgs vev insertions. In this section we will study only the effects coming from one Higgs insertion because the diagrams with three Higgs insertions will be suppressed due to the additional powers of the $\theta_{H}^{2} \equiv\left(\frac{v}{\sqrt{2} f_{h}}\right)^{2}$. The dominant contribution to the $Z_{C} b_{L} \bar{b}_{L}$ is shown on the Fig. 9. If we only consider individual contributions coming from these mixings, then the naive estimate of the $Z_{C} b_{L} \bar{b}_{L}$ coupling will be (we ignore the difference between $\theta_{H}$ and $\sin \theta_{H}$ )

$$
\sim g_{*}\left(\frac{1}{2}-c_{1}\right) \theta_{H}
$$

However, to get a more precise estimate, first let us look at the diagram (1) of Fig. 9, In this case intermediate gauge boson can be either $W_{L}^{3, \mathrm{KK}}$ or $W_{R}^{3, \mathrm{KK}}$ (they are the heavy gauge bosons of the generators $T_{L}^{3}$ and $T_{R}^{3}$ in two-site language). To analyze the coupling $h Z_{C, \mu} W_{L, R}^{3, \mathrm{KK}, \mu}$ we will 


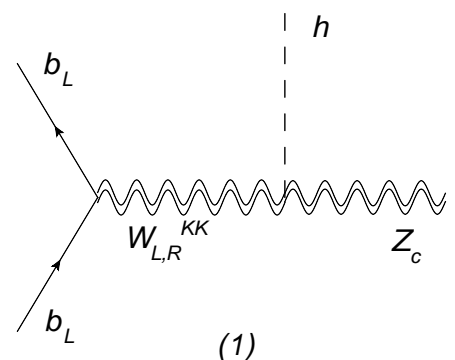

(1)

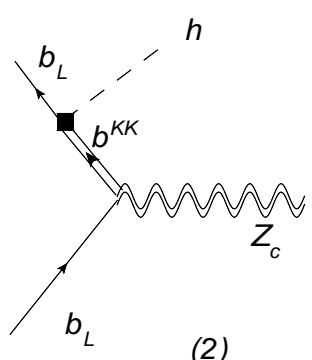

(2)

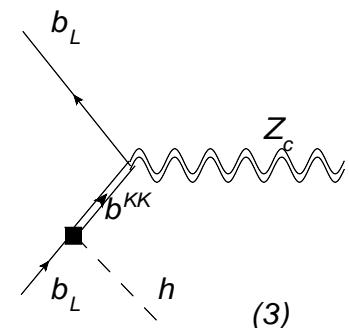

(3)

Figure 9: Diagrams contributing to the $Z_{c} \bar{b} b$ at $\frac{v}{f_{h}}$ order

use two-site approach, one can see that this coupling arises from the covariant derivative of the $\phi$ field $\left|D_{\mu} \phi\right|^{2}$ (see Eq. (6) ). Performing commutation relations one can show that the couplings of $Z_{C}$ to $W_{L}^{3, \mathrm{KK}}$ and $W_{R}^{3, \mathrm{KK}}$ have opposite signs, so the effective coupling of $Z_{C}$ to the SM fermions coming from diagram(1) of Fig. 9 is proportional to $\left(T_{L}^{3}-T_{R}^{3}\right)$ of the $b_{L}$ field. But we know that to overcome the constraint from the shift in $Z \bar{b}_{L} b_{L}$ coupling, $b_{L}$ should be in such representation of $S U(2)_{L} \times S U(2)_{R}$ to have $T_{L}^{3}=T_{R}^{3}$ [19]. This means that in realistic models of PGB Higgs, contribution to $Z_{c} b_{L} \bar{b}_{L}$ coupling from diagram (1) of Fig. 9 is zero.

Now let us look on the diagrams (2) and (3) of Fig.9. Note that $h$ and $Z_{C}$ are accompanied by the generators $T_{c}^{4}$ and $T_{c}^{3}$ of $S O(5)$ respectively. Therefore in this case coupling of the $Z_{C}$ to the $\mathrm{SM} b_{L}$ is proportional to

$$
\bar{b}_{L}\left\{T_{c}^{3}, T_{c}^{4}\right\} b_{L}
$$

But one can see that in our model SM $b_{L}$ lives mostly in the $\mathbf{5}$ of the $\mathrm{SO}(5)$, then one can check by direct calculation that

$$
\xi_{b}^{\dagger} \cdot A^{\dagger} \cdot\left\{T_{c}^{3}, T_{c}^{4}\right\} \cdot A \cdot \xi_{b}=0
$$

where $\xi_{b}^{T}=\left(0,0,0, \psi_{b}, 0\right)$. This concludes our analysis of the suppression of the $Z_{C} b_{L} \bar{b}_{L}$ couplings.

\section{References}

[1] L. Randall and R. Sundrum, Phys. Rev. Lett. 83, 3370 (1999) arXiv:hep-ph/9905221.

[2] T. Gherghetta and A. Pomarol, Nucl. Phys. B 586, 141 (2000) arXiv:hep-ph/0003129.

[3] H. Davoudiasl, S. Gopalakrishna, E. Ponton and J. Santiago, arXiv:0908.1968 [hep-ph].

[4] K. Agashe, A. Delgado, M. J. May and R. Sundrum, JHEP 0308, 050 (2003) arXiv:hep-ph/0308036. 
[5] R. Contino, Y. Nomura and A. Pomarol, Nucl. Phys. B 671, 148 (2003) arXiv:hep-ph/0306259.

[6] See, for example, M. Carena, E. Ponton, J. Santiago and C. E. M. Wagner, Nucl. Phys. B 759, 202 (2006) arXiv:hep-ph/0607106 and Phys. Rev. D 76, 035006 (2007) arXiv:hep-ph/0701055

[7] R. Contino, T. Kramer, M. Son and R. Sundrum, JHEP 0705, 074 (2007) arXiv:hep-ph/0612180.

[8] A. D. Medina, N. R. Shah and C. E. M. Wagner, Phys. Rev. D 76, 095010 (2007) arXiv:0706.1281 [hep-ph]].

[9] M. Serone, arXiv:0909.5619 [hep-ph].

[10] Y. Hosotani, Phys. Lett. B 126, 309 (1983) and Phys. Lett. B 129, 193 (1983).

[11] K. Agashe, R. Contino and A. Pomarol, Nucl. Phys. B 719, 165 (2005) arXiv:hep-ph/0412089];

[12] Y. Hosotani, K. Oda, T. Ohnuma and Y. Sakamura, Phys. Rev. D 78, 096002 (2008) [Erratumibid. D 79, 079902 (2009)] [arXiv:0806.0480 [hep-ph]].

[13] J. M. Maldacena, Adv. Theor. Math. Phys. 2, 231 (1998) [Int. J. Theor. Phys. 38, 1113 (1999)] arXiv:hep-th/9711200]; S. S. Gubser, I. R. Klebanov and A. M. Polyakov, Phys. Lett. B 428, 105 (1998) arXiv:hep-th/9802109]; E. Witten, Adv. Theor. Math. Phys. 2, 253 (1998) arXiv:hep-th/9802150.

[14] H. Georgi and D. B. Kaplan, Phys. Lett. B 136, 183 (1984); B 145, 216 (1984);

D. B. Kaplan, H. Georgi and S. Dimopoulos, Phys. Lett. B 136, 187 (1984);

H. Georgi, D. B. Kaplan and P. Galison, Phys. Lett. B 143, 152 (1984);

M. J. Dugan, H. Georgi and D. B. Kaplan, Nucl. Phys. B 254, 299 (1985).

[15] N. Arkani-Hamed, M. Porrati and L. Randall, JHEP 0108, 017 (2001) arXiv:hep-th/0012148;

R. Rattazzi and A. Zaffaroni, JHEP 0104, 021 (2001) arXiv:hep-th/0012248.

[16] R. Contino, L. Da Rold and A. Pomarol, Phys. Rev. D 75, 055014 (2007) arXiv:hep-ph/0612048.

[17] M. Carena, A. D. Medina, B. Panes, N. R. Shah and C. E. M. Wagner, Phys. Rev. D 77, 076003 (2008) arXiv:0712.0095 [hep-ph]]. R. Contino and G. Servant, JHEP 0806, 026 (2008) [arXiv:0801.1679 [hep-ph]]; J. Mrazek and A. Wulzer, arXiv:0909.3977 [hep-ph]; for a general 
analysis of LHC signals of such top quark "partners", see J. A. Aguilar-Saavedra, JHEP 0911, 030 (2009) [arXiv:0907.3155 [hep-ph]].

[18] M. V. Chizhov and G. Dvali, arXiv:0908.0924 [hep-ph].

[19] K. Agashe, R. Contino, L. Da Rold and A. Pomarol, Phys. Lett. B 641 (2006) 62 arXiv:hep-ph/0605341.

[20] S. J. Huber, Nucl. Phys. B 666, 269 (2003) arXiv:hep-ph/0303183; K. Agashe, G. Perez and A. Soni, Phys. Rev. D 71, 016002 (2005) arXiv:hep-ph/0408134]; S. Casagrande, F. Goertz, U. Haisch, M. Neubert and T. Pfoh, JHEP 0810, 094 (2008) arXiv:0807.4937 [hepph]]; M. Blanke, A. J. Buras, B. Duling, S. Gori and A. Weiler, JHEP 0903, 001 (2009) arXiv:0809.1073 [hep-ph]]; M. Blanke, A. J. Buras, B. Duling, K. Gemmler and S. Gori, JHEP 0903, 108 (2009) arXiv:0812.3803 [hep-ph]]; M. E. Albrecht, M. Blanke, A. J. Buras, B. Duling and K. Gemmler, arXiv:0903.2415 [hep-ph]; A. J. Buras, B. Duling and S. Gori, JHEP 0909, 076 (2009) arXiv:0905.2318 [hep-ph]].

[21] C. Csaki, A. Falkowski and A. Weiler, JHEP 0809, 008 (2008) arXiv:0804.1954 [hep-ph]].

[22] K. Agashe, arXiv:0902.2400 [hep-ph].

[23] O. Gedalia, G. Isidori and G. Perez, arXiv:0905.3264 [hep-ph].

[24] A. L. Fitzpatrick, G. Perez and L. Randall, arXiv:0710.1869 [hep-ph]; M. C. Chen and H. B. Yu, Phys. Lett. B 672, 253 (2009) arXiv:0804.2503 [hep-ph]]; G. Perez and L. Randall, JHEP 0901, 077 (2009) arXiv:0805.4652 [hep-ph]]; C. Csaki, C. Delaunay, C. Grojean and Y. Grossman, JHEP 0810, 055 (2008) arXiv:0806.0356 [hep-ph]]; J. Santiago, JHEP 0812, 046 (2008) arXiv:0806.1230 [hep-ph]]; C. Csaki, A. Falkowski and A. Weiler, arXiv:0806.3757 [hep-ph]; C. Csaki, G. Perez, Z. Surujon and A. Weiler, arXiv:0907.0474 [hep-ph]; M. C. Chen, K. T. Mahanthappa and F. Yu, arXiv:0909.5472 [hep-ph].

[25] H. Davoudiasl, J. L. Hewett and T. G. Rizzo, Phys. Rev. D 68, 045002 (2003) arXiv:hep-ph/0212279; M. Carena, E. Ponton, T. M. P. Tait and C. E. M. Wagner, Phys. Rev. D 67, 096006 (2003) arXiv:hep-ph/0212307; M. S. Carena, A. Delgado, E. Ponton, T. M. P. Tait and C. E. M. Wagner, Phys. Rev. D 68, 035010 (2003) arXiv:hep-ph/0305188; M. S. Carena, A. Delgado, E. Ponton, T. M. P. Tait and C. E. M. Wagner, Phys. Rev. D 71, 015010 (2005) arXiv:hep-ph/0410344.

[26] P. McGuirk, G. Shiu and K. M. Zurek, JHEP 0803, 012 (2008) arXiv:0712.2264 [hep-ph]]; G. Shiu, B. Underwood, K. M. Zurek and D. G. E. Walker, Phys. Rev. Lett. 100, 031601 (2008) 
arXiv:0705.4097 [hep-ph]]; A. Falkowski and M. Perez-Victoria, arXiv:0806.1737 [hep-ph]; B. Batell, T. Gherghetta and D. Sword, arXiv:0808.3977 [hep-ph]; A. Delgado and D. Diego, Phys. Rev. D 80, 024030 (2009) arXiv:0905.1095 [hep-ph]]; S. Mert Aybat and J. Santiago, arXiv:0905.3032 [hep-ph].

[27] private communication with A. Falkowski and M. Son.

[28] T. E. W. Group [CDF Collaboration and D0 Collaboration], arXiv:0908.2171 [hep-ex].

[29] T. Han, H. E. Logan and L. T. Wang, JHEP 0601, 099 (2006) arXiv:hep-ph/0506313.

[30] K. Agashe et al., Phys. Rev. D 76, 115015 (2007) arXiv:0709.0007 [hep-ph]]; K. Agashe, S. Gopalakrishna, T. Han, G. Y. Huang and A. Soni, arXiv:0810.1497 [hep-ph].

[31] A. Falkowski, Phys. Rev. D 75, 025017 (2007) [arXiv:hep-ph/0610336].

[32] K. Agashe, A. Azatov and L. Zhu, Phys. Rev. D 79, 056006 (2009) [arXiv:0810.1016 [hep-ph]].

[33] J. Pumplin, D. R. Stump, J. Huston, H. L. Lai, P. M. Nadolsky and W. K. Tung, JHEP 0207, 012 (2002) arXiv:hep-ph/0201195].

[34] G. L. Bayatian et al. [CMS Collaboration], J. Phys. G 34, 995 (2007). G. Aad et al. [The ATLAS Collaboration], arXiv:0901.0512 [hep-ex].

[35] V. M. Abazov et al. [D0 Collaboration], Phys. Rev. Lett. 99, 191802 (2007) arXiv:hep-ex/0702005.

[36] T. Han, H. E. Logan, B. McElrath and L. T. Wang, Phys. Rev. D 67, 095004 (2003) arXiv:hep-ph/0301040.

[37] W. Y. Keung and G. Senjanovic, Phys. Rev. Lett. 50, 1427 (1983). 